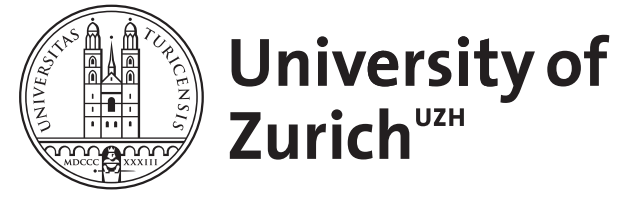

\title{
Zur Demut beim Lehren und Lernen
}

\author{
Negri, Silvia
}

DOI: https://doi.org/10.1515/9783110461770-008

Posted at the Zurich Open Repository and Archive, University of Zurich

ZORA URL: https://doi.org/10.5167/uzh-203501

Book Section

Published Version

Originally published at:

Negri, Silvia (2016). Zur Demut beim Lehren und Lernen. In: Speer, Andreas; Jeschke, Thomas. Schüler und Meister: Akten der 39. Mediävistentagung. Berlin: De Gruyter, 107-134.

DOI: https://doi.org/10.1515/9783110461770-008 


\title{
Zur Demut beim Lehren und Lernen
}

\author{
Silvia Negri (Freiburg im Breisgau)
}

\section{Demut, Meister, Schüler. Ur-Figuren}

Durch wiederkehrende Beispiele, Metaphern und Assoziationen lassen sich verschiedene Auffassungen der Demut im lateinischen Mittelalter rekonstruieren. Räumliche Bilder scheinen insbesondere dazu zu dienen, den bumilis und die humilitas zu repräsentieren. Häufig wird in den Darstellungen der Demut der bumilis als derjenige beschrieben, der an der Erde (bumus) teilhat, und nicht über seine Kapazitäten hinaus emporzuragen beansprucht, also derjenige, der seine Menschlichkeit (bumanitas) nicht übersteigt ${ }^{1}$.

In den Texten und sogar in der Ikonografie der humilitas tauchen verschiedene Topoi auf: die Treppe, welche Erde und Himmel verbindet, und auf der die Demut die erste, niedrigste Stufe darstellt; der Baum der Tugenden mit der Demut als dessen Wurzel; der Turm der Sitten, der auf der Demut basiert sowie der gesenkte Blick des Demütigen ${ }^{2}$. Um die Paarbegriffe der Niedrigkeit gegenüber der Höhe kreisen dann weitere antithetische Bilder. Die Selbstentleerung des Demütigen wird der Selbsterfüllung des Arroganten gegenübergestellt. Der Demütige wird als gefügig beschrieben und der Unterwerfung hingegeben.

Interessanterweise schließt sich die Topik der Demut wesentlich an den begrifflichen und semantischen Bereich der Lehre, mit den Figuren des Meisters und des Schülers, an. Die Ur-Figur des Christus als magister humilitatis, der dem

1 Referenzaufsätze zu den Ausdeutungen der humilitas sind A. Dihle, Demut, in: Reallexikon für Antike und Christentum, vol. 3, Stuttgart 1957, 735-778; P. Adnès, Humilité, in: Dictionnaire de spiritualité, vol. 6, Paris 1969, 1136-1187. Bekanntlich hat Gauthier ein wirkmächtiges Paradigma der Demut als charakteristischer Tugend des spätantiken Christentums und des Frühmittelalters skizziert, welche der magnanimitas der Antike entgegengesetzt war. Magnanimitas, die Großmut, wurde - laut Gauthiers These - zusammen mit Aristoteles im 13. Jahrhundert im akademischen Kontext wiederentdeckt, wodurch die Rolle der Demut etwas zurückgetreten ist. Dieses Paradigma werde ich weder thematisieren noch bewerten. Cf. R.-A. Gauthier, Magnanimité. L'idéal de la grandeur dans la philosophie païenne et dans la théologie chrétienne, Paris 1951. Es sei hier auch auf folgende Studie verwiesen: I. P. Bejczy, Cardinal Virtues in a Christian Context: The Antithesis between Fortitude and Humility in the Twelfth Century, in: Medioevo 31 (2006), 49-67.

2 Zu den Repräsentationen der bumilitas cf. e.g. A. Katzenellenbogen, Allegories of the Virtues and Vices in Mediaeval Art. From Early Christian Times to the Thirteenth Century, London 1939 [Neudruck Nendeln/Liechtenstein 1968] und C. Hourihane (ed.), Virtue \& Vice. The Personifications in the Index of Christian Art, Princeton, NJ 2000, bes. 230-239. 
Teufel, Urbild des Hochmutes, der superbia, Wurzel aller Sünde entgegengesetzt ist, diente im christlichen Mittelalter als theologische Grundlage für eine positive Konnotation der Demut als Tugend der Erlösung ${ }^{3}$. Vom Himmel zur Erde, von oben nach unten wurde mit dem Fall des Engels Luzifer und mit der Vertreibung von Adam und Eva aus dem Paradies der Ur-Pfad des Hochmuts beschrieben. Die Inkarnation, der Tod Gottes und die Auferstehung bilden hingegen die gegensätzlichen Pfade der höchsten Erniedrigung und der darauf folgenden höchsten Erhebung ${ }^{4}$.

Das christliche Ideal der Nachahmung Christi schloss auch die Notwendigkeit ein, die Demut zu praktizieren. Der Schüler des Christus-Meister sollte sich die bumilitas aneignen, die von Christus paradigmatisch verkörpert wurde. In einer Passage seines Buchs, De sancta virginitate' behauptete Augustinus, dass die ganze christliche Lehre (universa disciplina christiana) sich dem Hochmut widersetzt (militat) und dabei die bumilitas lehrt $(\text { docet })^{5}$. Diese Passage zur disciplina christiana zusammen mit der Lehre des bene vivere, die von Christus, dem Meister, seiner Kirche beigebracht wird, veranschaulicht paradigmatisch die Bedeutung der bumilitas als Hauptinhalt der christlichen Lehre schlechthin ${ }^{6}$. In dieser scheinen die Erlernbarkeit und die Lehrbarkeit ein weiteres wesentliches Merkmal der Darstellungen der humilitas zu bilden. Die Bescheidenheit kann am Beispiel

3 Cf. C. Casagrande/S. Vecchio, I sette vizi capitali. Storia dei peccati nel Medioevo (Saggi 832), Torino 2000, insbes. 3-33.

4 Cf. Augustinus, Enarrationes in Psalmos, 31, 2, edd. D. E. Dekkers/J. Fraipont (Corpus Christianorum. Series Latina 38), Turnhout 1956, 18, 43-49: „Via bumilitatis buius aliunde manat; a Christo venit. Haec via ab illo est, qui cum esset altus, bumilis venit. Quid enim aliud docuit bumilando se, factus oboediens usque ad mortem, mortem autem crucis? [...] Quid aliud docuit nisi hanc bumilitatem?" Zur augustinischen Auffassung der Demut cf. unter den neueren Publikationen T. Howe, Weisheit und Demut bei Augustinus, in: A. Goltz/A. Luther/H. Schlange-Schöningen (eds.), Gelehrte in der Antike. Alexander Demandt zum 65. Geburtstag, Köln-Weimar-Wien 2002, 219-236; C. Mayer, Humiliatio, Humilis, in: id. (ed.), Augustinus-Lexikon, vol. 3, Basel 2006, 443-456, mit sämtlichen bibliografischen Hinweisen; N. Baumann, Die Demut als Grundlage aller Tugenden bei Augustinus (Patrologia 21), Frankfurt 2009.

5 Cf. Augustinus, De sancta virginitate, 31, 31, ed. J. Zycha (Corpus Scriptorum Ecclesiasticorum Latinorum 41), Wien 1900, 268, 16-269, 3: „Mensura bumilitatis cuique ex mensura ipsius magnitudinis data est: cui est periculosa superbia, quae amplius amplioribus insidiatur. Hanc sequitur invidentia, tanquam filia pedissequa: eam quippe superbia continuo parit, nec unquam est sine tali prole atque comite. Quibus duobus malis, boc est superbia et invidentia, diabolus est. Itaque contra superbiam, matrem invidentiae, maxime militat universa disciplina christiana. Haec enim docet bumilitatem, qua et adquirat et custodiat caritatem".

6 Cf. Augustinus, De disciplina christiana, 1, ed. R. Vander Plaetse (Corpus Christianorum. Series Latina 46), Turnhout 1969, 1-11: „Locutus est ad nos sermo dei, et depromptus est ad exhortationem nostram, dicente scriptura: accipite disciplinam in domo disciplinae. Disciplina, a discendo dicta est: disciplinae domus, est ecclesia christi. Quid ergo bic discitur, vel quare discitur? Qui discunt? A quo discunt? Discitur bene vivere. Propter hoc discitur bene vivere, ut perveniatur ad semper vivere. Discunt christiani, docet christus. [...] Omnes in domo disciplinae sumus, sed multi nolunt habere disciplinam, et, quod est perversius, nec in domo disciplinae volunt habere disciplinam" und ibid., 14, 379-385: „Quis est enim magister qui docet? Non qualiscumque homo, sed apostolus. Plane apostolus, et tamen non apostolus. An vultis, inquit, experimentum eius accipere, qui in me loquitur Christus? Christus est qui docet; cathedram in caelo babet, ut paulo ante dixi. Schola ipsius in terra est, et schola ipsius corpus ipsius est. Caput docet membra sua, lingua loquitur pedibus suis. Christus est qui docet: audimus, timeamus, faciamus". 
des außergewöhnlichen Lebens Christi gelehrt und so zum Vorbild werden. Sie kann auch auf eine besondere Weise der Übung erworben werden.

Ein ständig wiederkehrender Topos der lateinischen Literatur in Bezug zur bumilitas ist ebenso das Vorschreiben einer Reihe von Akten und Verhaltensweisen, durch die die Demut des Körpers, das heißt der Gesten und der Haltung, und die Bescheidenheit des Geistes in einem Prozess der aufsteigenden Verinnerlichung der tugendhaften Disposition gewonnen wird. Dieses Motiv taucht besonders in jenen literarischen Gattungen auf, in denen die Nachahmung Christi als Lebensform und sogar als Lebensregel angeordnet wird. Die bumilitas wird als Grundlage für das eigene sowie das gemeinschaftliche Leben vorgeschrieben, indem sie die Gehorsamkeit gegenüber Gott und den anderen, den Meistern und den Mitbrüdern, lehrt. In den monastischen Regeln erhält daher die Bescheidenheit eine Schlüsselrolle als Garant der Gesetze, die von dem Abbas vorgeschrieben werden, um die Meister-Figur Christi nachzubilden, und die von den Schülern verinnerlicht werden sollen ${ }^{7}$. Dadurch dass sie die eigene Mangelhaftigkeit anerkennt, realisiert sich die bumilitas durch äußerliche und innerliche Unterordnung und immer neuen Gehorsam ${ }^{8}$. In epistemischer Hinsicht artikulierte sich tatsächlich der humilitas-Topos in der Anerkennung der menschlichen Schwäche und konstitutiven Unwissenheit. Dem Lob der bewussten Ignoranz schloss sich somit das Bekenntnis der Notwendigkeit an, stetig belehrt zu werden und sich dabei als demütige Schüler zu zeigen.

Als Eigenschaft des discipulus wurde die Bescheidenheit in einer breiten Palette mittelalterlicher Quellen gepriesen, die zwar auf diverse Entstehungskontexte, Rezipienten und Intentionen hinweisen, sich aber gemeinsamer Figuren und rekurrierender Auffassungen bedienten. Die Verbindung zwischen Disziplin, Lehre (Lehrer und Schüler betreffend) und Demut bildet das Kernobjekt der vorliegenden Untersuchung. Mit Blick auf den Entstehungskontext und die Peri-

\footnotetext{
7 Mit dem monastischen Erziehungswesen, das mit dem griechischen Modell der Philosophie verglichen wird, und zwar mit Blick auf den Begriff der docilitas, beschäftigt sich G. Jeanmart, Généalogie de la docilité dans l'Antiquité et le Haut Moyen Âge (Philosophie de l'éducation), Paris 2007, insbes. 93-247. Zur Geschichte des Gehorsamsgelübdes als juristisches Verhältnis in den monastischen Regeln und zu seinen Vorläufern im Versprechen der oboedientia cf. bekanntlich C. Capelle, Le voeu d'obéissance des origines au XII ${ }^{\mathrm{e}}$ siècle. Étude juridique (Bibliothèque d'histoire du droit et droit romain 2), Paris 1959. Zur rebellio und inoboedientia im Mönchtum, die sich dem Laster der superbia anschließen, cf. e. g. G. Melville, Der Mönch als Rebell gegen gesetzte Ordnung und religiöse Tugend. Beobachtungen zu Quellen des 12. und 13. Jahrhunderts, in: id. (ed.), De ordine vitae. Zu Normvorstellungen, Organisationsformen und Schriftgebrauch im mittelalterlichen Ordenswesen (Vita regularis. Ordnungen und Deutungen religiosen Lebens im Mittelalter 1), Münster 1996, 153-186.

$8 \mathrm{Zu}$ den Eigentümlichkeiten der früheren monastischen Regeln als literarischer Gattung cf. A. de Vogüé, Les règles monastiques anciennes $(400-700)$ (Typologie des sources du Moyen Âge occidental 46), Turnhout 1985. In Bezug auf die für die Regel charakteristische Überschneidung zwischen regula und vita, Norm und Leben, cf. E. Coccia, Regula et vita. Il diritto monastico e la regola francescana, in: Medioevo e Rinascimento 20/n.s. 17 (2006), 97-147 mit besonderem Bezug auf den Franziskanerorden, und G. Agamben, Altissima povertà. Regole monastiche e forma di vita, Vicenza 2011.
} 
ode diverser Primärtexte werde ich versuchen, einige Kernelemente in jenen Diskursen herauszuarbeiten, in denen der humilitas eine positive Konnotation im Bildungskonzept zukam und in denen die Bescheidenheit nicht nur dem discipulus, sondern auch dem magister empfohlen oder sogar verordnet wurde. Als paradigmatische Beispiele werden in diesem Kontext zunächst einige monastische Regeln und Texte genannt, die im Mönchtum entstanden sind. Über die Identifizierung der humilitas mit der disciplina als der zu praktizierenden Haltung des Unterworfenen in der ,Regula Magistri' und in der ,Regula Benedicti‘ hinaus wird somit die Verknüpfung zwischen Zucht und Lehre untersucht. Außerdem werden zwei Werke des Hugo von Sankt Viktor in Betracht gezogen, die eine ausführliche Überlegung zur Demut des discipulus an der Schnittstelle von praktischem und kontemplativem Bereich bieten. Die Verbindung zwischen der Lehre aus magistraler Sicht und der Bescheidenheit wird dann hauptsächlich anhand von Schriften analysiert, die im universitären Umfeld im Laufe des 13. Jahrhunderts verfasst wurden. Dabei werden entgegengesetzte Auffassungen des Profils eines akademischen Magisters kurz skizziert, um zu überprüfen, wie ähnliche Figuren und Paarbegriffe (praesumptio gegen bumilitas) $\mathrm{zu}$ unterschiedlichen Zwecken eingesetzt wurden. Schließlich werde ich auf die Verordnungen der Mäßigkeit in einigen universitären Statuten aus dem 14. und 15. Jahrhundert hinweisen.

Meine Textauswahl ist von der Suche nach gemeinsamen und paradigmatischen Figuren der praktizierten oder zu praktizierenden Demut geleitet. Das daraus resultierende Panorama beansprucht folglich keine Vollständigkeit, doch soll eine gewisse Repräsentativität in den gewählten Bildern der Demut gesucht werden. Auch auf die Gefahr hin, hier zu vereinfachen, könnte man die genannten Quellen als Repräsentanten (auf jeweils unterschiedliche Weisen und mit unterschiedlichen Konnotationen) pädagogischer Diskurse auffassen, die für konkurrierende mittelalterliche Systeme und Erziehungsmodelle typisch sind: das Kloster, die Schule, die Universität. Das Ideal der Bescheidenheit wird in diesen Bildungsbereichen mit Hilfe der analysierten Topik untersucht, die dem Auseinandergehen der Konzepte von sittlicher Lebensführung einerseits und Wissenschaftspraktizierung andererseits entspricht. Das Motiv der Bescheidenheit als Haltung des Schülers, der sich dem Meister als einem Übergeordneten unterwirft und in die Gemeinschaft von Schülern aufgenommen wird, kehrt in den gewählten Quellen mit einer gewissen Beharrlichkeit wieder, wie ich zeigen werde.

Der Reichtum von Bedeutungen, die schon seit der klassischen Antike die hier betroffenen semantischen Felder kennzeichneten - man denke paradigmatisch an discipulus/disciplina -, verlangt ein stetig sich erneuerndes Verständnis der von den Quellen verwendeten und von mir kommentierten Begrifflichkeit. Es wird gelegentlich auf hilfreiche lexikografische Studien verwiesen. Ich werde mich auch nicht nur auf die Wortanalyse beschränken, sondern vielmehr auf plausible Vergleichskontexte hindeuten. 


\section{Humilitas vel disciplina: das Mönchtum}

Gehorsam, Schweigsamkeit und Bescheidenheit schrieb im 6. Jahrhundert die ,Regula‘ des Benedikt von Nursia vor ${ }^{9}$. Als Grundlage für das Zusammenleben in der monastischen Gemeinschaft galt die Unterwerfung den Mitbrüdern und dem Abbas gegenüber; sie wurde als Zeichen und gleichzeitig Ergebnis der Liebe zu Gott dargestellt ${ }^{10}$. Als erster Schritt auf der Treppe der Demut wurde im 5. Kapitel der ,Regula' die oboedientia befohlen, aufgrund derer der Schüler (discipulus) den Anweisungen seines Meisters (magister) - als Auswirkung der göttlichen Furcht - eifrig und bereitwillig dadurch folgt, dass er seinen eigenen Willen aufgibt ${ }^{11}$. Ebenso wurde für den discipulus bestimmt, die taciturnitas auszuüben, denn es ist dem Meister das Lehren und dabei das Reden eigen, wohingegen der Schüler zuhören und daher Stillschweigen bewahren soll ${ }^{12}$. Wenn es für den discipulus notwendig ist, zu Wort zu kommen, so fährt die Regel fort, weil er den Oberen, um etwas bitten will, dann ,,soll es in aller Demut und ehrfürchtiger Unterordnung erbeten werden" ${ }^{13}$. Die reverentia ist die charakteristische Haltung

9 Cf. Benedicti Regula. Editio altera et correcta, insbes. 5-7, ed. R. Hanslik (Corpus Scriptorum Ecclesiasticorum Latinorum 75), Wien 1977, 38-57.

10 Unter den Studien zur Regel sei an dieser Stelle auf A. de Vogüé, La communauté et l'abbé dans la Règle de Saint Benoît, Paris 1960, insbes. Kap. IV, verwiesen. In unserem Zusammenhang interessante Bemerkungen zur oboedientia gegenüber dem Abbas als „Lehrer“, zunächst bei Cassianus und in der ,Regula Magistri‘, finden sich ibid., 275-276: „Dans la perspective de Luc 10, 16, le supérieur apparaît avant tout comme un maitre de doctrine évangelique. Le bienfait qu'on attend de lui est d'enseigner la loi divine [...] Cette nécéssité d'un ,docteur' est le premier fundament de l'obéissance monastique [...] L'obéissance s'impose donc tout d'abord comme une nécessité pédagogique. Qui veut apprendre un art doit se mettre à bonne école. L'art spiritual ne fait pas exception à cette loi “.

11 Benedicti Regula, 5, 1-10, ed. Hanslik (nt. 9), 38-39: „Primus bumilitatis gradus est oboedientia sine mora. Haec convenit his, qui nibil sibi a Christo carius aliquid existimant; propter servitium sanctum, quod professi sunt, seu propter metum gehennae vel gloriam vitae aeternae, mox aliquid imperatum a maiore fuerit, ac si divinitus imperetur, moram pati nesciant in faciendo. De quibus dominus dicit: Obauditu auris oboedivit mibi. Et item dicit doctoribus: Qui vos audit, me audit. Ergo bii tales relinquentes statim, quae sua sunt, et voluntatem propriam deserentes mox exoccupatis manibus et, quod agebant, imperfectum relinquentes vicino oboedientiae pede iubentis vocem factis sequuntur. Et veluti uno momento praedicta magistri iussio et perfecta discipuli opera in velocitate timoris dei ambae res communiter citius explicantur, quibus ad vitam aeternam gradiendi amor incumbit“. Die oboedientia wird laut Capelle zum ersten Mal in der ,Regula Benedicti“ (58, $17-18,149)$ Gegenstand eines monastischen Versprechens, zusammen mit der stabilitas und der conversatio morum, cf. Capelle, Le voeu d'obeissance (nt. 7), 91 (zu den Bezügen zwischen der ,Regula Benedicti“ und der ,Regula Magistri“ 94-98). Capelle unterstrich die ,, geistliche“ Bedeutung jenes offiziellen Aktes der Unterwerfung und des Gehorsams den Übergeordneten in der Gemeinschaft (servitium sanctum) gegenüber, der einer „situation juridique spéciale“ entspricht, in der man sich zunächst Gott innerlich unterwirft, cf. ibid., 92.

12 Cf. Benedicti Regula, 6, 6, ed. Hanslik (nt. 9), 42: „Nam loqui et docere magistrum condecet, tacere et audire discipulum convenit".

13 Cf. Benedicti Regula, 6, 7, ed. Hanslik (nt. 9), 42: „Et ideo si qua requirenda sunt a priore, cum omni bumilitate et subiectione reverentiae requirantur". In seiner, Expositio“ zur ,Regula' Benedikts kommentierte der Benediktinermönch Smaragdus von St. Mihiel die Passage auf folgende Weise: „Qui enim a priore consilium salutis et verba doctrinae requirit, cum omni bumilitate vel subiectione quaerere debet, quia summa monachi virtus humilitas est, summum vitium est superbia. Tunc autem se quisque monachum 
des discipulus gegenüber dem Meister und sie gründet explizit in der Unterwerfung und in der Bescheidenheit.

Die Demut (humilitas) spielt in der ,Regula' eine besonders zentrale Rolle für denjenigen, der durch Übung und Praktizierung der zu erreichenden Tugend und durch Erniedrigung seiner selbst sich der wahren, erlösenden Hoheit öffnet. Das biblisch geprägte Vorbild der Leiter (scala) der Demut, auf welcher Gehorsam und Ehrfurcht die erste Stufe bilden, und die Erde und Himmel in einer wechselseitigen Beziehung verbindet, bildete die spekulative, theologische und geistliche Voraussetzung, um den Wert des Dienstes der Ehrfurcht, der Unterordnung, der Loslösung vom eigenen Willen und die Anerkennung der eigenen Mangelhaftigkeit zu untermauern ${ }^{14}$. Dabei wurde eine Anthropologie der Demut geboten: In der Leiter-Metapher, welche die Demut veranschaulicht, werden der Leib und die Seele des Menschen als Holme beschrieben, in die Gott verschiedene, aufzusteigende Stufen der Demut gesetzt hat:

„Latera enim eius scalae dicimus nostrum esse corpus et animam, in qua latera diversos gradus bumilitatis vel disciplinae evocatio divina ascendendo inseruit" $" 15$.

Sowohl in Bezug auf den Körper als auch auf die Seele soll der Mensch die Demut ausüben, um sich selbst als Mensch zu realisieren. Besonders bemerkenswert ist hier die terminologische Überlappung von humilitas und disciplina. Die Bescheidenheit wird als Eigenschaft des discipulus schlechthin verstanden. Mehr noch: Demut ist die disciplina selber.

In einem 1934 veröffentlichten Aufsatz analysierte Henri-Irénée Marrou die Verwendungen der Wörter doctrina und disciplina insbesondere vom 3. bis zum 6. Jahrhundert, das heißt von der christlichen Spätantike bis hin zur ,Regula“ Benedikts, die zu dem gemeinsamen semantischen Feld der erteilten und der empfangenen Lehre gehören ${ }^{16}$. Gemäß dem lexikografischen Bericht von Mar-

indicet, quando se minimum existimaverit, etiam cum maiora virtutum opera gesserit. Semper enim conscientia servi dei bumilis debet esse et tristis, scilicet ut per humilitatem non superbiat, et per utilem merorem cor ad lasciviam non dissolvat", cf. Smaragdi Abbatis Expositio in Regulam S. Benedicti, edd. A. Spannagel/P. Engelbert (Corpus consuetudinum monasticarum 8), Siegburg 1974, 160, 27 - 33.

14 Cf. Benedicti Regula, 7, 5-8, ed. Hanslik (nt. 9), 43-44: „Unde, fratres, si summae bumilitatis volumus culmen adtingere et ad exaltationem illam caelestem, ad quam per praesentis vitae bumilitatem ascenditur volumus velociter pervenire, actibus nostris ascendentibus scala illa erigenda est, quae in somnio Iacob apparuit, per quam ei descendentes et ascendentes angeli monstrabantur. Non aliud sine dubio descensus ille et ascensus a nobis intelligitur nisi exaltatione descendere et humilitate ascendere. Scala vero ipsa erecta nostra est vita in saeculo, quae bumiliato corde a domino erigatur ad caelum". Der timor und die memoria der Gebote Gottes werden mit der ersten Stufe der scala der bumilitas 7, 10-13, ibid., 45-46 gleichgesetzt: „Primus itaque bumilitatis gradus est, si timorem dei sibi ante oculos semper ponens oblivionem omnino fugiat et semper sit memor omnia, quae praecepit deus [...] Et custodiens se omni hora a peccatis et vitiis, id est cogitationum, linguae, manuum, pedum vel voluntatis propriae, sed et desideria carnis, aestimet se homo de caelis a Deo semper respici omni hora et facta sua omni loco ab aspectu divinitatis videri et ab angelis omni hora renuntiari".

15 Benedicti Regula, 7, 9, ed. Hanslik (nt. 9), 44.

16 Cf. H.-I. Marrou, „Doctrina“ et „disciplina“ dans la langue des Pères de l’Église, in: Archivum latinitatis medii aevi 9 (1934), 5-25. Cf. auch W. Dürig, Disciplina. Eine Studie zum Bedeutungsumfang des Wortes in der Sprache der Liturgie und der Väter, in: Sacris Erudiri 4 (1952), 245 279. Über die Bezüge zwischen disciplina als Fach zu den Parallelbegriffen von Kunst (ars) und 
rou weist die vielfache Verwendung des Wortes disciplina in der Benediktsregel auf die körperliche und geistige Bestrafung hin, der sich der Mönch unterwerfen muss; im Allgemeinen ist die Verwendung des Wortes disciplina auf die Bedeutungen von Unterwerfung, Autorität, Gehorsam, korrektem Verhalten und Einübung zurückzuführen ${ }^{17}$. Demut und Unterwerfung sowie Demut und strafbare Haltung fallen somit zusammen. Sie bringen die Verbindung von dem Gebot Gottes und der Loslösung von dem eigenen menschlichen Willen zum Ausdruck, die aus der Anerkennung der eigenen Schwäche und Abhängigkeit erfolgt $^{18}$.

Die Verbindung von humilitas und disciplina tauchte schon in der ,Regula Magistri' auf, die als unmittelbare Quelle der ,Regula‘ Benedikts diente. Das zehnte Kapitel der ,Regula Magistri“ wurde der Demut unter der Überschrift „De bumilitate fratrum, qualis debeat esse vel quibus modis adquiritur vel quomodo adquisita servatur" gewidmet, in welchem die Stufen der himmlischen Leiter der Demut beschrieben wurden, die der discipulus hinaufsteigen soll ${ }^{19}$. Unter der Überschrift „Quo ordine tacentes fratres aliquas interrogationes faciant abbati" hatte der Autor der ,Regula“ die Motive der Verbeugung und der Schweigsamkeit des discipulus dem Meister gegenüber entwickelt ${ }^{20}$.

Wissenschaft (scientia) hinaus untersuchte Marie-Dominique Chenu weitere Anwendungen des Konzepts der Disziplin im 12. und 13. Jahrhundert, cf. M.-D. Chenu, Notes de lexicographie philosophique médiévale. Disciplina, in: Revue des sciences philosophiques et théologiques 25 (1936), 686-692 [Neudruck in: id., Studi di lessicografia filosofica medievale, ed. G. Spinosa (Lessico Intellettuale Europeo 86), Firenze 2001, 93-100]. Ebenso widmete 1957 Jean Leclerq in dem ,Dictionnaire de Spiritualité‘ dem Wort disciplina einen Artikel. In seiner Rekonstruktion beschränkte sich Leclerq auf die „, geistliche Sphäre“, cf. J. Leclerq, Disciplina, in: Dictionnaire de Spiritualité, vol. 3, Paris 1957, 1291-1302.

17 Cf. ibid., 20-21, 24-25.

18 Interessanterweise unterschied Smaragdus in seinem im 9. Jahrhundert verfassten Kommentar die Konzepte der bumilitas und der disciplina dadurch, dass er die Bescheidenheit als Wächterin der Disziplin der Gelehrsamkeit beschrieb. Derjenige, der aufgrund der bumilitas weich und sanft geworden sei, wird aufgrund der disciplinae eruditio geschult: „Gradus isti ideo bumilitatis et disciplinae dicuntur, quia istae ambae virtutes pulcherrime sibi invicem sociantur; quia quem bumilitas mansuetum, disciplinae eruditio componit et facit doctum, et quem bumilitas tranquillum et mitem, disciplinae eruditio facit providum et sapientem. Et quia scriptum est, Neminem diligit deus nisi eum qui cum sapientia commoratur', necesse est ut bumilitati disciplina iungatur, quia eruditio disciplinae custos est bumilitatis, et bumilitas temperatio est eruditionis", cf. Smaragdi Abbatis Expositio in Regulam S. Benedicti, edd. Spannagel/Engelbert (nt. 13), 166, 10-17.

19 Cf. Regula Magistri/La règle du Maître, 10, ed. A. de Vogüé, vol. 1 (Sources chrétiennes 105), Paris 1964, 418-444. Cf. dazu den Kommentar A. de Vogüés, ibid., 95 sqq., demzufolge das Ziel des Befehls zu Gehorsam, Schweigsamkeit und Demut im „Kampf gegen die Sünde“ besteht, cf. insbes. 96.

20 Cf. Regula Magistri, 9, ed. de Vogüé (nt. 19), 406, 1-410, 37: „Quo ordine tacentes fratres aliquas interrogationes faciant abbati. Respondit Dominus per magistrum: Cum de malis tamque bonis eloquiis freno taciturnitatis constringuntur discipuli et licentiae eorum aditus praesentis magistri custodia observantur, cum necessariae utilitati eorum aliquae interrogationes advenerint, adbuc clauso ore et signato tacitae gravitatis sigillo, stantes ante maiorem inclinato bumilitatis capite, clausum in silentio os clave benedictionis aperiant. Quod si semel dictae benedictioni ad postulandum eloquium adbuc non respondeat magistri permissio, iterata bumiliatio capitis et benedictione sola iterum repetita denuo ab abbate postuletur licentia. Quod si nec sic ab eo responsum fuerit, curvato item in humilitate discipulo, iam ipse frater se tunc demum removeat, ne aut nimius ei aut 
Ebenso beschrieb der Benediktiner Peter von Celle in seinem 1179 verfassten Traktat ,De disciplina claustrali‘ die Demut als Säule der klösterlichen Disziplin. In dem einführenden Widmungsbrief schrieb Petrus die poenalis disciplina als notwendiges Mittel gegen die korrumpierte geistige Freiheit vor. Dabei wurde die bumilitas als Werkzeug präsentiert, durch welches das Hochmütige und das Ungezähmte gebeugt werden können ${ }^{21}$. Die vom Christus-Lehrer in der weltlichen Schule gelehrte Disziplin wird im Traktat als das Leben gemäß den Geboten Gottes bezeichnet ${ }^{22}$. Der Ursprung der klösterlichen Disziplin liegt zudem in der Kreuzigung als Erfahrung einer höchsten Erniedrigung ${ }^{23}$. Offensichtlich erforderte eine Lebensform, die sich als irdisches, mangelhaftes Spiegelbild eines höheren Lebens versteht, die Bestimmung von Regeln, die immer wieder eingeübt werden müssen. Die Demut bezeichnete hierbei die Offenheit für das aufgenommene Gebot, welche aus der Anerkennung der Autorität des Gebotes selber stammt. Was erlernt wird, ist eine Praktik, eine Haltung, eine Form des Lebens oder vielmehr eine Form des Erlernens. Disciplina ist zugleich Inhalt und Form des Gebotes, welches eine kontinuierliche Selbsterniedrigung erfordert. Die stetig erneuerte Anerkennung der eigenen Mangelhaftigkeit übt sich in einem Verhalten des Körpers und der Seele unmittelbar aus.

appareat inprobus, et suo operi redditus adbuc gerat personam tacentis, ut in ipsa bumilitate taciturnitatis extimet semetipsum $\langle i d e o\rangle$ indignum indicatum ad loquendum abbatis indicio, aut forte ad probationem inveniendae bumilitatis putet sibi discipulus taciturnitatis ideo fuisse non aperta clausura. Ideo enim ipsam solam benedictionem denuo diximus repetiri, ut cum sola est et sine alio eloquio, tamen reservata diu taciturnitas conservetur, cum denuo repetitur et post ipsam mox a nolente disceditur, adbuc conservari probata humilitas sentiatur. Nam ideo repetitio discipuli reoffertur magistro, ut non solum propter spiritalem probationem ad inveniendam bumilitatem discipuli diu possit tacere magister, sed ne et corporaliter per cogitatum animo occupatus, vultus eius aliis cogitationibus obstupescens vocem praecantis discipuli surdis auribus praetermittat, et ne ipsum magistrum per nimiam et inportunam humilitatem in irae cogat vitio declinare et inportuni bumilitas in inpedimento scandali deputetur". Zur Schweigsamkeit cf. e.g. A. M. Orselli, L’equilibrio tra silenzio e parola nella comunicazione monastica tardoantica (IV-VI secolo), in: Silenzio e parola nella patristica: XXXIX Incontro di studiosi dell'antichità cristiana, Roma, 1-8 maggio 2010 (Studia ephemeridis Augustinianum 127), 109-118. Zu Betrachtungen de peccato linguae aus dem 12. und 13. Jahrhundert cf. C. Casagrande/S. Vecchio, I peccati della lingua. Disciplina ed etica della parola nella cultura medievale, Roma 1987.

21 Cf. Peter von Celle, De disciplina claustrali/L'école du cloître, epist. dedic., ed. G. de Martel (Sources chrétiennes 240), Paris 1977, 96, 4-11: „Libertas animi nisi ex proprio arbitrio et adiuncto limosi corporis consortio infatuata fuisset, nequaquam poenali disciplina quae plicat, planat et ligat, indiguisset. Plicat erecta, planat aspera et ligat lasciva. Plicat indomita, planat inaequalia, ligat dissoluta. Plicat animum, corpus planat, utrumque ligat. Plicat bumilitate, planat aequitate, ligat religione. Plicat conversione, planat confessione, ligat professione".

22 Cf. Peter von Celle, De disciplina claustrali, I, ed. de Martel (nt. 21), 120, 21 - 25: ,Quid est autem nostra disciplina, nisi vivere secundum mandata Dei? Iesus vero magister magnus et bonus, cuius cathedra est in coelo et scola in mundo, in evangelio quasi in antidotario quae fecit et docuit".

23 Cf. Peter von Celle, De disciplina claustrali, VI, ed. de Martel (nt. 21), 160, 1-11: „Sed quid haec ad claustralem disciplinam? Plane tota forma claustralis disciplinae emanavit de cruce. Sicut enim Christus exaltatus a terra suspensus est in cruce, expoliatus vestimentis suis, et sic distensus ut dinumerarentur omnia ossa eius, ligno sic affixus est ut nullum membrum praeter linguam moveret, quam retinuerat ad orandum et matrem dilecto discipulo commendandam, sic claustralis qui vere claustralis est debet se totum crucifigere cum vitiis et concupiscentiis, debet se a terra sustollere, sicut scriptum est: Sustollam te super altitudines terrae". 
Trotz zeitlicher Distanz und ungeachtet eines anderen Kontexts fungierte diese Topik als eine Konstante in der literarischen Gattung der klösterlichen Regel und klösterlichen Anweisungen ${ }^{24}$. In der franziskanischen Regel wird die Demut als Korrelat für die Armut und Geduld sowie für den geistlichen Frieden verordnet ${ }^{25}$. Es findet sich paradigmatisch eine wesentliche Verbindung von bumilitas und disciplina im Traktat mit dem Titel ,Speculum disciplinae', der Bonaventura zugeschrieben wurde, sehr wahrscheinlich aber im 13. Jahrhundert von dem Franziskaner Bernhard von Bessa verfasst wurde ${ }^{26}$. Im ersten Teil des Traktates, der sich der Propädeutik der klösterlichen Disziplin als Einübung einer Lebensform widmet, wird die Aneignung der bumilitas zusammen mit dem Vermeiden der innerlichen und äußerlichen praesumptio als unvermeidbare Wurzel für die Fähigkeit der Disziplin charakterisiert ${ }^{27}$.

\section{An der Schnittstelle zwischen Wissen und Lebensführung}

Die Metapher von Siegel und Wachs wurde vor Bernhard von Bessa im 12. Jahrhundert von Hugo von Sankt Viktor in seinem ,De institutione novitiorum' benutzt, um die Novizen auf die guten Beispiele von vita und conversatio hinzuweisen, die als Ausdruck der göttlichen Form nachgeahmt werden müs$\operatorname{sen}^{28}$. Die menschliche Fähigkeit, sich von dem Guten und damit auch von Gott

24 Weitere Beispiele von klösterlichen Anweisungen aus dem ausgehenden Mittelalter, in denen dieselbe Topik vorkommt, könnten genannt werden; cf. e. g. Thomas von Kempen, Libellus de disciplina claustralium, ed. M. I. Pohl (Opera omnia 2), Freiburg i. Br. 1904, 265-319, insbes. 4 („De oboedientia humilis subditi erga praelatum suum“), 282-287.

25 Cf. Franz von Assisi, Regula non bullata, in: E. Menestò/S. Brufani (eds.), Fontes Francescani (Medioevo Francescano. Testi 2), Assisi 1995, 183-212, insbes. Kap. 9 und 17 und Regula bullata, ibid., 169-181, insbes. Kap. 3, 6, 10, 12. Zum Kapitel 10 der ,Regula bullata und insbesondere zur Ausdeutung der Aufforderung „et non current nescientes litteras, litteras discere“ cf. P. Maranesi, Nescientes litteras. L'ammonizione della regola francescana e la questione degli studi nell'ordine (sec. XIII-XVI) (Bibliotheca Seraphico-Capuccina 61), Roma 2000.

26 Cf. dazu B. Roest, A History of Franciscan Education (c. 1210-1517) (Education and Society in the Middle Ages and Renaissance 11), Leiden-Boston-Köln, 2000, insbes. 246-247.

27 Cf. Bernhard von Bessa, Speculum disciplinae, in: Bonaventurae Opera omnia, vol. 8, Quaracchi 1898, 583-622, hier 7 („De disciplina in generali“), 591: „Dicto de praeparatoriis ad disciplinam et aliquibus eius effectibus incidenter, sequitur, ut, de ipsa plenius exprimentes, quae vetustate deposita animoque contra hostis insidias stabilito, super radices bumilitatis consurgit, primo in generali quid sit, quae sit eius utilitas, qualiter ad eius perveniatur notitiam et de ipsius custodia, deinde in speciali dicamus.“ Cf. auch 5 (,De praesumptione tam in re quam in signo vitanda“), ibid., 587, 588 und 589: „Praesumptionem interiorem et exteriorem summopere fugiant [...] Exterioris praesumptionis et superbiae notam in omni actione sua, gestu et verbo summo studio fugiant, quae bumilitatis sunt in omnibus amplexantes [...] Praesumptionem ergo evitat prudens humilitas; praesumit tamen interdum humiliter viscerosa et provida caritas [...] Reprehensibilis maxime in iuvene praesumptio, quem magis bumilitas stabilit, verecundia ornat, venustat simplicitas, timor insignit et aptum efficit disciplinae, cuius initium est".

28 Cf. Hugo von Sankt Viktor, De institutione novitiorum, 7, in: L' oeuvre de Hugues de SaintVictor. Texte latin par H. B. Feiss/P. Sicard, traduction française par D. Poirel/H. Rochais, introduction, notes et appendices par D. Poirel, vol. 1, Turnhout 1997, 40, 340-348: "Quare putatis, fratres, uitam et conuersationem bonorum imitari precipimur, nisi ut per eorum imitationem ad noue uite similitudinem reformemur? In ipsis siquidem similitudinis Dei forma expressa est, et idcirco, cum eis per 
prägen zu lassen - wie weiches Wachs, das die Form aufnimmt -, erfordert die Demut als Zugrundeliegendes, die von der Selbstüberhebung befreit ${ }^{29}$. Bescheidenheit, Schweigsamkeit und Offenheit werden zudem von Hugo im Traktat als Eigenschaften desjenigen Novizen gepriesen, der in der Schule der Tugenden belehrt werden möchte, und sich dabei auf das Zuhören einstellen muss ${ }^{30}$. Der bumilitas und Sanftheit werden die Streitsüchtigkeit, die Redseligkeit und der Neid entgegengestellt ${ }^{31}$. Der Mahnung, die eigene Zunge vor dem Überfluss leerer Wörter zu hüten, folgt die Aufforderung zu mensura und modum sowie die Anregung, eher auditores denn doctores zu sein ${ }^{32}$. Der Bescheidenheit im Sprechen entspricht nämlich die Scharfsinnigkeit im Verstehen ${ }^{33}$. Um sich von der Wahrheit überzeugen zu lassen und dabei ,vere discipuli veritatis“ zu sein, so Hugo von Sankt Viktor weiter, muss man die doctrina veritatis von den Belehrten demütig

imitationem imprimimur, ad eiusdem similitudinis imaginem nos quoque figuramur. Sed sciendum est quia, sicut cera, nisi prius emollita fuerit, formam non recipit, sic et homo quidem per manum actionis aliene ad formam uirtutis non flectitur, nisi prius per humilitatem ab omni elationis et contradictionis rigore moliatur". Cf. auch Bernhard von Bessa, Speculum disciplinae, 3 (nt. 27), 583: „Denique, ,quoniam, ut ait Hugo, sicut cera, nisi prius emollita fuerit, formam non recipit, sic et homo quidem ad formam virtutis non flectitur, nisi ab omni elationis et contradictionis rigore per humilitatem molliatur; necesse habent qui disciplinae cupiunt moribus informari, ut bumilitatis radicem in corde flegere studeant et tumorem propriae voluntatis, praesumptionis et irriverentiae vitia, quae a superbia procedere solent, tam in re quam in signo vigilantius caveant, ut bumilitas ancillae more locum praeparet disciplinae."

29 Hugo von Sankt Viktor und der Viktoriner Schule sind neuerdings zwei Sammelbände gewidmet worden, die ich hier als allgemeine Referenzstudien erwähnen möchte: cf. D. Poirel (ed.), L'école de Saint-Victor de Paris. Influence et rayonnement du Moyen Âge à l'époque moderne. Colloque international du C.N.R.S. pour le neuvième centenaire de la fondation $(1108-2008)$ tenu au Collège des bernardins à Paris le 24-27 septembre 2008 (Bibliotheca Victorina 22), Turnhout 2010 und Ugo di San Vittore. Atti del XLVII Convegno storico internazionale, Todi, 10-12 ottobre 2010 (Atti dei convegni del Centro italiano di studi sul basso Medioevo, Accademia Tudertina 24), Spoleto 2011.

$30 \mathrm{Zu}$ der Viktoriner Auffassung der Bildung als Ausgleich von litterae und mores cf. C. S. Jaeger, Humanism and Ethics at the School of St. Victor in the Early Twelfth Century, in: Mediaeval Studies 55 (1993), $51-79$.

31 Cf. Hugo von Sankt Viktor, De institutione novitiorum, 6, edd. Feiss/Sicard (nt. 28), 36, $268-$ 37, 271; 38, 288-296: „Quoniam igitur ad scholam virtutum erudiendi acceditis, scire debetis contentiones uerborum nullo modo deinceps ad uos pertinere, quia spiritualis doctrine studium non litigantes sed auscultantes requirit. [...] Nam quia rixe et contentiones uerborum de pestifera semper radice oriantur, dubium non est quoniam, nisi cor prius intrinsecus per elationem intumesceret, nequaquam lingua foris a custodia bumilitatis sue in contumeliam uerborum se relaxaret. Sed dum superbe pre aliis sapientes videri volumus, erubescimus uel nostram ignorantiam ab aliis argui, uel aliorum sapientiam quasi ad nostram depressionem approbari. Sicque nonnunquam contra conscientiam nostram uel nostrum errorem impudenter defendimus, uel alterius ueritatem malitiose impugnamus".

32 Cf. Hugo von Sankt Viktor, De institutione novitiorum, 6, edd. Feiss/Sicard (nt. 28), 38, 311314: „Proinde, fratres carissimi, linguam uestram a superfluitate non solum inanium uerborum restringite, sed in ipsis etiam que recta sunt mensuram et modum tenete, et in collatione sanctorum magis auditores quam doctores esse eligite“. Zur disciplina in locutione in diesem Traktat cf. Casagrande/Vecchio, I peccati della lingua (nt. 20), $77-83$.

33 Cf. Hugo von Sankt Viktor, De institutione novitiorum, 6, edd. Feiss/Sicard (nt. 28), 38, 314316: „Os clausum et aures apertas babete, et quanto estis ad loquendum modestiores, tanto estote ad intelligendum sagaciores". 
aufnehmen, die Einfachen nicht verachten, die jene doctrina „verwalten“, sich als niedrig in der scientia Dei einschätzen und sich nicht schämen, von allen anderen Menschen beziehungsweise Mitbrüdern etwas zu lernen ${ }^{34}$. Die bescheidene Gesinnung öffnet also den discipulus, damit er die doctrina empfangen kann. Aber gerade die Lehre (doctrina) zusammen mit der ratio, dem exemplum, der meditatio der Heiligen Schrift sowie der inspectio operum ac morum suorum ist für den Autor einer der Wege, die zur Erlangung des Wissens bezüglich des guten Lebens (recte vivendi scientia) führen ${ }^{35}$.

Im programmatischen Prolog zu der Schrift hatte Hugo in Anlehnung an die Psalmen 118 und 17 die disciplina - insbesondere die disciplina virtutis, die eingeübt wird, um sich die Tugenden anzueignen - als Tor zur Glückseligkeit des ewigen Lebens beschrieben ${ }^{36}$. Die göttliche Disziplin (disciplina Dei) wird nämlich als Voraussetzung für das Gutsein dargestellt und setzt ihrerseits den Besitz der scientia discretionis, des Unterscheidungsvermögens, voraus ${ }^{37}$. Der Weg zu Gott besteht also in dem Wissen, durch das die Disziplin erlangt wird: Disziplin, die zum Gutsein führt, und Gutsein, welches zur Glückseligkeit geleitet ${ }^{38}$. Der Novize kann sich der disciplina, das heißt dem Gebot Gottes, nur unterwerfen und dabei die disciplina als Selbstmäßigung ausüben, wenn er die Regel des guten Lebens kennt.

Ralf Stammberger hat unterstrichen, dass für Hugo „,die ,scientia“ den theoretischen Aspekt der Bildung darstellt“, da sie „die Quellen des Wissens und die Fähigkeit zur ,discretio“" miteinschließt, „die erst zum rechten Gebrauch der

34 Cf. Hugo von Sankt Viktor, De institutione novitiorum, 6, edd. Feiss/Sicard (nt. 28), 40, 323336: „Restat ergo, fratres, ut, si nere discipuli neritatis esse cupitis, tales nos studeatis exhibere quibus neritas facile persuaderi possit, quia et ipsa doctrina ueritatis tales auditores amat, qui eam et a sapientibus exbibitam bumiliter suscipiunt, a simplicioribus quoque administratam arroganter non contemnunt; qui in scientia Dei, hoc est in peritia bene agendi, cunctos prudentiores et sapientiores quam se esse existimant, nec erubescunt magistros habere omnes a quibus discere possunt quod ignorant; qui bene dicta omnium libenter approbant, errores uero alienos, quantum ad se pertinere cognouerunt, pro tempore student uel modeste corripere uel prudenter dissimulare; qui denique studium scientiae morum disciplina exornant et mansuetudinem ac modestiam mentis sue, non solum in custodia oris, sed etiam in habitu et gestu corporis sui demonstrant".

35 Cf. Hugo von Sankt Viktor, De institutione novitiorum, 1, edd. Feiss/Sicard (nt. 28), 22, $62-$ 66.

36 Cf. Hugo von Sankt Viktor, De institutione novitiorum, prol., edd. Feiss/Sicard (nt. 28), 18, $9-$ 14: „Ad beatitudinem autem nemo uenire potest, nisi per uirtutem, et uirtus non alio modo veraciter apprehenditur, nisi disciplina virtutis non negligenter custodiatur. V sus enim discipline ad uirtutem animum dirigit, uirtus autem ad beatitudinem perducit. Ac per hoc exercitium discipline esse debet uestra inchoatio, uirtus perfectio, praemium uirtutis eterna beatitudo".

37 Cf. Hugo von Sankt Viktor, De institutione novitiorum, prol., edd. Feiss/Sicard (nt. 28), 18, 19-21 und 20, 30-33: „Disciplina namque Dei in finem corrigit, quia, plene et perfecte hominem reformans per virtutem, ad beatitudinem perducit [...] Postremo, quia uidit neminem seruare posse disciplinam boni operis, qui non habeat scientiam uere discretionis, hanc quoque sibi dari quasi quoddam fundamentum et principium sancte conuersationis postulanit".

38 Cf. Hugo von Sankt Viktor, De institutione novitiorum, prol., edd. Feiss/Sicard (nt. 28), 20, 55-22, 60: „Via ad ipsum sunt scientia, disciplina, bonitas; per scientiam itur ad disciplinam, per disciplinam itur ad bonitatem, per bonitatem itur ad beatitudinem. Hoc est propter quod vobiscum loqui cepimus, ut, quantum Dominus dederit, de hac via qua ad ipsum itur uos instruamus". 
,disciplina“ befähigt" ${ }^{\text {"39 }}$. Hierbei ist die scientia mit dem innerlichsten, intimen Bereich der geistlichen Übung, die disciplina hingegen mit der äußerlichen Dimension des Körperlichen, der Gesten und des beobachtbaren guten Benehmens verbunden. Denn „Ziel des mit ,disciplina“ bezeichneten praktischen Moments der Bildung ist die Bestimmung des Körpers durch den Geist. Allerdings bedarf zuvor der Geist selbst der Disziplinierung durch Einübung in ein geordnetes Verhalten" ${ }^{40}$. Beide Momente - scientia und disciplina - sind aber ohnehin tiefgehend von deren praktischem Ziel her bestimmt, das heißt von dem Gutsein und darüber hinaus von der höchsten Glückseligkeit. In der gesamten Bildungsauffassung von ,De institutione novitiorum' drückt sich tatsächlich die scientia in der Einsicht dessen aus, was man tun soll, um ein gutes, tugendhaftes Leben zu führen. Es handelt sich also um ein auf die irdische Praxis ausgerichtetes Wissen, welches wiederum zu einem weiteren Lebensmodus - dem ewigen Leben anleitet. Hierzu wirkt die Demut als eine der Voraussetzungen für die Aufnahme

39 Cf. R. M. W. Stammberger, „Via ad ipsum sunt scientia, disciplina, bonitas“. Theorie und Praxis der Bildung in der Abtei Sankt Viktor im zwölften Jahrhundert, in: R. Berndt/M. Lutz-Bachmann/R. M. W. Stammberger (eds.), „Scientia“ und „disciplina“. Wissenstheorie und Wissenschaftspraxis im 12. und 13. Jahrhundert (Erudiri Sapientia 3), Berlin 2002, 91-126, hier insbes. $112-113$.

40 Cf. ibid., 113. Jaeger hat dem Lemma ,disciplina im Kontext von ,De institutione novitiorum und des ,Didascalicon“ einen Teil seines schon zitierten Aufsatzes gewidmet, cf. Jaeger, Humanism and Ethics at the School of St. Victor (nt. 30), 57-61. Der Wissenschaftler merkte an, dass: „Discipline“ is the central ethical/pedagogical conception of the De institutione novitiorum [...] It designates both the content and the process of ethical training [...] Throughout Hugh's work ,discipline' is the process of learning virtue, the central activity of ethical education", ibid., 57. In Anlehnung an die Definition von disciplina, die in Hugos ,De institutione novitiorum“ 10 vorkommt („Disciplina est conuersatio bona et honesta, cui parum est malum non facere, sed studet etiam in biis que bene agit per cuncta irreprehensibilis apparere. Item disciplina est membrorum omnium motus ordinatus et dispositio decens in omni habitu et actione", cf. Hugo von Sankt Viktor, De institutione novitiorum, 10, edd. Feiss/Sicard [nt. 28], 48, 451-455), versuchte Jaeger, den Unterschied zwischen dieser Konzeption von Disziplin und der monastischen Idee der disciplina zu erhellen, cf. Jaeger, Humanism and Ethics at the School of St. Victor (nt. 30), 58-59: „This definition expressly limits its meaning to an etiquette of conduct, bearing, control, and governance of the body [...]. Hugh is speaking an ethical language that he does not share with monastic tradition or with the traditions of canons regular, where disciplina as a process of ethical training ordinarily meant the teaching and learning of the rule, or simply the rule itself, and had nothing like the profile it has in the De institutione novitiorum"; parallel dazu lokalisierte Jaeger die Auffassung der Viktoriner eher in einer klassischen Tradition, die sich in den Höfen und Kathedralschulen weiter ausbreitete, cf. ibid., 59. Zu disciplina und „, geste“ in Hugo cf. bekanntlich auch J.-C. Schmitt, La raison des gestes dans l'occident médiéval, Paris 1990, insbes. 174-205. In seinem Kommentar zu der Passage räumte hingegen Poirel die Verwendung des Begriffes von disciplina in ,De institutione novitiorum' in der monastischen Tradition ein, cf. Hugo von Sankt Viktor, De institutione novitiorum, edd. Feiss/Sicard (nt. 28), 107, nt. 61. Cf. auch R. Baron, Hugues de Saint-Victor lexicographie, in: Cultura neolatina 16 (1956), 109-145 und D. Romagnoli, ,Disciplina est conversatio bona et honesta': anima, corpo e società tra Ugo di San Vittore ed Erasmo da Rotterdam, in: P. Prodi (ed.), Disciplina dell'anima, disciplina del corpo e disciplina della società tra medioevo ed età moderna (Annali dell'Istituto storico italo-germanico 40), Bologna 1994, 507 537. 
des Wissens, und zwar jenes Wissens, das von außen, von einem Lehrer, erworben werden soll. Somit ist die Demut Grundlage der Disziplin schlechthin, welche ihrerseits als Joch des Hochmuts gekennzeichnet wird.

Aus einer Synthese von theoretisch und praktisch bestimmten Faktoren besteht ebenso die Konzeption der Bildung, die Hugo von Sankt Viktor in seinem ,Didascalicon de studio legendi' vertrat. Eine ausbalancierte Mischung von mores und scientia setzte der Viktoriner Meister in seinem Werk beim lector, also beim Studierenden voraus ${ }^{41}$. Bekanntlich präsentierte sich das um 1127 verfasste Werk als ein enzyklopädisches Handbuch zur lectio als einem Weg neben der meditatio, mittels dessen man unterwiesen wird, um die scientia zu erlangen ${ }^{42}$. Die disciplina des lector wird dabei als die sittliche Haltung gegenüber dem Lernen und der Wissensaufnahme verstanden, die sich in einer Reihe von Elementen verwirklicht, die man teilweise auch in den schon ausgeführten Regelungen wiederfinden kann.

Stammberger hat weiterhin auf die grundlegende Einheit des Bildungsprogramms Hugos von Sankt Viktor hingewiesen, das sich nicht nur an die Mitglieder des Ordens richtete, sondern das Hugo bekanntlich auch in einer offenen Schule durchführen ließ. Stammberger hat dabei die wiederkehrenden Motive aus ,De institutione novitiorum' und ,Didascalicon“ hervorgehoben. Sowohl für das ,interne“ als auch für das „externe“ Bildungswesen schlug Hugo eine Erziehungsauffassung vor, in der scientia und disciplina die Leitbegriffe darstellen ${ }^{43}$. Für das ,Didascalicon', das sich die Systematik und die Einteilung der weltlichen Wissenschaften, die Methoden und Praktiken der lectio artium einerseits, die $\mathrm{Na}$ tur und Verfahrensweise der lectio divinarum scripturarum andererseits zum Gegenstand machte, ist „der Bezug zwischen der Lektüre und der Lebensordnung““ das Eigentümlichste ${ }^{44}$. Ausschlaggebend ist in diesem Kontext die Rolle der Demut als Garant der Disziplin.

Mit einem berühmten Aphorismus, den auch Johannes von Salisbury in seinem ,Policraticus', VII, 13 benutzte, und der laut Aussage des Johannes auf Bernhard von Chartres zurückgeht, führte Hugo von Sankt Viktor das Kapitel im dritten Buch seines ,Didascalicon“ ein, das er dem Lemma disciplina widmete:

„Sapiens quidam cum de modo et forma discendi interrogaretur: ,Mens' inquit , bumilis, studium quaerendi, vita quieta, scrutinium tacitum, paupertas, terra aliena, haec reserare solent multis obscura legendi “ 45 .

41 Cf. Hugo von Sankt Viktor, Didascalicon de studio legendi. Übersetzt und eingeleitet von T. Offergeld (Fontes Christiani 27), Freiburg e. a. 1997. Ich werde auch auf die kritische Edition des Textes verweisen, cf. Hugo von Sankt Viktor, Didascalicon de studio legendi. A Critical Text, ed. C. H. Buttimer (The Catholic University of America. Studies in Medieval and Renaissance Latin 10), Washington, DC 1939.

42 Cf. Hugo von Sankt Viktor, Didascalicon, praefatio, ed. Offergeld (nt. 41), 106 (ed. Buttimer [nt. 41], 2, 9-11): „Duae praecipue res sunt quibus quisque ad scientiam instruitur, videlicet lectio et meditatio, e quibus lectio priorem in doctrina obtinet locum, et de hac tractat liber iste dando praecepta legendi".

43 Cf. Stammberger, „Via ad ipsum sunt scientia, disciplina, bonitas“ (nt. 39), 114.

44 Cf. ibid., 118.

45 Hugo von Sankt Viktor, Didascalicon, III, 12, ed. Offergeld (nt. 41), 250, 1-5 (ed. Buttimer [nt. 41], 61, 10-14). Im selben Kapitel betonte Hugo die enge Verknüpfung zwischen mores 
Der erste Maßstab für Methode und Form des discere besteht in der mens humilis. In dem Kapitel ,De humilitate erkannte Hugo die Demut als Anfang der Disziplin (principium disciplinae) und als Grundlage des sittlichen Erlernens ${ }^{46}$. Die disciplina ist ihrerseits eine der Voraussetzungen für die Studierenden, zusammen mit natürlicher Begabung und Übung ${ }^{47}$. Die Ausübung der Disziplin fördert den Schüler darin, ,daß er ein lobenswertes Leben führt, indem er moralisches Verhalten und Wissenschaftlichkeit vereint "48. Vor dem Hintergrund dieser Verflechtung von Wissen und Sitten werden insbesondere drei Elemente von Hugo als Zeichen einer demütigen Haltung beim discere aufgelistet: 1. alle Wissenschaften und alle Schriften hochzuschätzen; 2. sich nicht zu schämen, von anderen etwas zu lernen; 3. den anderen nicht $\mathrm{zu}$ verachten, wenn man schon belehrt ist ${ }^{49}$.

Neben dem Motiv der prudentia, die den Studierenden dazu führt, alles zu lesen und sich anzuhören, dabei aber ,keine Schrift, keine Person, keine Lehre zu verachten“, trat bei Hugo von Sankt Viktor wiederum das Bekenntnis des eigenen Unwissens auf ${ }^{50}$. Das Resultat der Anerkennung der eigenen Ignoranz führt zu einer wohlwollenden Stellung gegenüber allen Formen des Wissens:

„Ab omnibus libenter disce quod tu nescis, quia bumilitas commune tibi facere potest quod natura cuique proprium fecit. Sapientior omnibus eris, si ab omnibus discere volueris. Qui ab omnibus accipiunt, omnibus ditiores sunt" ${ }^{* 51}$.

In der Auffassung Hugos von Sankt Viktor ist das Lernen-Wollen mit dem bumilitas-Aspekt verbunden sowie mit der Idee, dass das Aufnehmen von Wissen (accipere) sich mit einer Offenheit in Bezug auf den Lehrenden verbindet. Diese

und scientia, cf. ibid., 6-11 (ed. Buttimer [nt. 41], 61, 14-19): „Audierat, puto, quod dictum est: ,Mores ornant scientiam', et ideo praeceptis legendi, praecepta quoque vivendi, adiungit, ut et modum vitae suae et studii sui rationem lector agnoscat. Illaudabilis est scientia quam vita maculat impudica. Et idcirco summopere cavendum ei qui quaerit scientiam, ut non negligat disciplinam".

46 Cf. Hugo von Sankt Viktor, Didascalicon, III, 13, ed. Offergeld (nt. 41), 250, 12 (ed. Buttimer [nt. 41], 61, 22).

47 Cf. Hugo von Sankt Viktor, Didascalicon, III, 6, ed. Offergeld (nt. 41), 238, 18-19 (ed. Buttimer [nt. 41], 57, 6): „Tria sunt studentibus necessaria: natura, exercitium, disciplina“.

48 Cf. Hugo von Sankt Viktor, Didascalicon, III, 6, ed. Offergeld (nt. 41), 238, 21 - 240, 1, deutsche Übersetzung 239-241 (ed. Buttimer [nt. 41], 57, 9-11).

49 Cf. Hugo von Sankt Viktor, Didascalicon, III, 13, ed. Offergeld (nt. 41), 250, $12-17$ (ed. Buttimer [nt. 41], 61, 22-62, 1): „Principium autem disciplinae bumilitas est, cuius cum multa sint documenta, haec tria praecipue ad lectorem pertinent: primum, ut nullam scientiam, nullam scripturam vilem teneat, secundum, ut a nemine discere erubescat, tertium, ut cum scientiam adeptus fuerit, ceteros non contemnat".

50 Cf. Hugo von Sankt Viktor, Didascalicon, III, 13, ed. Offergeld (nt. 41), 252, 18-22, deutsche Übersetzung 253 (ed. Buttimer [41], 62, 20-21): „Prudens igitur lector omnes libenter audit, omnia legit, non scripturam, non personam, non doctrinam spernit. Indifferenter ab omnibus quod sibi deesse videt quaerit, nec quantum sciat, sed quantum ignoret, considerat". Zu Wesen, Praktik und Zweck des Lesens im ,Didascalicon` cf. D. Poirel, Prudens lector. La pratique des livres et de la lecture selon Hugues de Saint-Victor, in: Cahiers de recherches médiévales et humanistes 17 (2009), 209-226.

51 Hugo de Sancto Victore, Didascalicon, III, 13, ed. Offergeld (nt. 41), 254, 9-13 (ed. Buttimer [nt. 41], 63, 4-7). 
wiederum erfolgt aus dem Bekenntnis der eigenen Unwissenheit. Der gute Student soll dabei mansuetus (sanftmütig), diligens (aufmerksam) und sedulus (eifrig) sein, „damit er von allen bereitwillig lerne“"52.

\section{Der Meister als Schüler \\ Mangel an Wissen und Besitz einer Wissenschaft}

In einem Text mit dem Titel ,De disciplina scolarium', der wahrscheinlich in der ersten Hälfte des 13. Jahrhunderts verfasst wurde, werden ebenso Gefügigkeit des Geistes, Aufmerksamkeit im Zuhören, eifrige Übung und wohlwollende Haltung in der Wissensaufnahme als charakteristisch für den sich gut benehmenden Studierenden beschrieben ${ }^{53}$. Mindestens bis zum 15. Jahrhundert wurde die anonyme Schrift Boethius zugeschrieben. Die große Anzahl der dazu verfassten Kommentare, der von ihr abhängigen pädagogischen Traktate und die Verwendung vonseiten mittelalterlicher Autoren deutet auf einen großen Erfolg des Werkes hin ${ }^{54}$. Als Entstehungskontext ist laut der Editorin des Textes, Olga Weijers, die Pariser Universität, genauer die Ausführungen zu dem Leben der Studenten und Magister dort zu identifizieren ${ }^{55}$. Mit dem Werk Hugos von Sankt Viktor teilt der anonyme Traktat die praktische Zielsetzung, wenn er pädagogische Anweisungen erteilt ${ }^{56}$. Bezüglich der disciplina der Schüler tritt im Traktat das Konzept der subiectio, der Unterwerfung, auf:

„Visis scolarium rudimentis et virtutum incrementis nunc de eorum subiectione erga magistratus breviter est ordinandum, quoniam qui non novit se subici, non noscet se magistrari. Miserum autem est eum magistrum fieri, qui numquam novit se discipulum esse. Debet autem discipuli subieccio in tribus consistere: in attencione, benivolencia et docilitate. Docilis ingenio, attentus exercicio, benivolus

52 Cf. Hugo von Sankt Viktor, Didascalicon, III, 13, ed. Offergeld (nt. 41), 256, 19-258, 8 (ed. Buttimer [nt. 41], 64, 6-16): „Bonus enim lector bumilis debet esse et mansuetus, a curis inanibus et voluptatum illecebris prorsus alienus, diligens et sedulus, ut ab omnibus libenter discat, numquam de scientia sua praesumat, perversi dogmatis auctores quasi venena fugiat, diu rem pertractare antequam iudicet discat, non videri doctus, sed esse quaerat, dicta sapientium intellecta diligat, et ea semper coram oculis quasi speculum vultus sui tenere studeat. Et si qua forte obscuriora intellectum eius non admiserint, non statim in vituperium prorumpat, ut nibil bonum esse credat, nisi quod ipse intelligere potuit. Haec est bumilitas disciplinae legentium".

53 Cf. Pseudo-Boethius, De disciplina scolarium, ed. O. Weijers (Studien und Texte zur Geistesgeschichte des Mittelalters 12), Leiden-Köln 1976.

54 Cf. O. Weijers, Introduction, ibid., insbes. 16-34.

55 Cf. ibid., 8-11.

56 Cf. Pseudo-Boethius, De disciplina scolarium, prol., ed. Weijers (nt. 53), 93, 2-4; 93, 16-94, 3: „Vestra novit intencio de scolarium disciplina compendiosum postulare tractatum - utinam compendiosum a spiritus mei parvitate [...] In primis prima, puerorum oblectamina sunt prelibanda. Secundo secunda, videlicet adultorum elacio qualiter magistratui est subicienda et discipline connectenda. Tercio de eorum elacione reprimenda, exemplorum commendationem distinctive componendo. Quarto de scolarium sagaci provisione eorumque graduali stacione. Quinto qualiter scolarium sincera ac venerabilis devocio ad magisterium est proferenda. Sexto de magistrancium norma divisioneque trina statuque erga subiectos modoque docendi precognito". 
animo. Attentus, inquam, ad audiendum, docilis ad intelligendum, benivolus ad retinendum. Ista vero tria ad perfeccionem currunt permutatim" $" 57$.

Interessanterweise tritt hier zu den in den vorangehenden Paragrafen genannten Motiven die Idee hinzu, dass die Unterwerfung nicht nur die Voraussetzung dafür ist, belehrt (magistrari) zu werden, sondern auch dafür, ein guter Meister zu werden. Nur der gute Schüler kann zu einem guten Lehrer werden; der eigentliche Meister ist ein bewusster Schüler. Selbst wenn im Kontext der Beschreibung der submissio des Schülers das semantische Feld der humilitas als solches nicht auftaucht, kehrt in der Tat das Adverb bumiliter in der Sektion des Traktates wieder, die den Eigenschaften eines guten magister gewidmet ist. Jeder doctor - schreibt der Traktat vor - soll gebildet (eruditus), friedlich, sanft (mansuetus), hart (rigidus), erfahren (antiquus), nicht hochmütig (non arrogans) sein. Die mansuetudo wird der arrogantia entgegengesetzt und der Hochmut der Bescheidenheit im Lehren ${ }^{58}$. Eine pädagogische Verpflichtung scheint den guten Magister darin festzulegen, die Lehre, die er empfangen hat, demütig und wohlwollend weiterzuvermitteln. Dazu soll der Blick des Lehrers nicht bei sich selber und bei seiner Gelehrsamkeit verweilen, sondern vielmehr zurück auf die Quelle seines Wissens gerichtet sein ${ }^{59}$. Der erfolgreiche Wissenstransfer setzt das Bewusstsein des Lehrers voraus, dass die von ihm erworbene scientia aus einem vorangehenden Akt des Lernens stammt.

In diesem Kontext lässt sich kurz das ,Compendium de virtute humilitatis“ des (Pseudo-)Bonaventura nennen, in dem die Demut mit dem geistigen $\mathrm{Zu}-$ stand des discipulus identifiziert wird. Von der Demut, definiert als ,recta consideratio sui et suorum defectuum ac periculorum et comparatio suae modicitatis ad magnitudinem omnium", wird im Traktat ausgesagt, dass sie sich eher als discipula denn als magistra verstanden wissen möchte, da sie sich mehr an die göttlichen Aussagen als an die eigene Vernünftigkeit oder die menschlichen Meinungen halten will ${ }^{60}$.

57 Pseudo-Boethius, De disciplina scolarium, 2, ed. Weijers (nt. 53), 99, 8-16. In jener Sektion seines Traktates ,De modo addiscendi', welche der subiectio des discipulus gewidmet wurde, führte Gilbert von Tournai die pseudo-boethianische Autorität an, cf. Gilbert von Tournai, De modo addiscendi, 3, ed. E. Bonifacio (Pubblicazioni del Pontificio Ateneo Salesiano. Testi e studi sul pensiero medioevale 1), Torino 1953, 105-106: „Primo ergo subiectio est necessaria. Nam paribus passibus ambulare debent scholarium rudimenta et virtutum incrementa. De hac ergo subiectione dicit Boethius: ,Qui non novit se subici non noscet se magistrari. Miserum autem est eum fieri magistrum qui se non novit esse discipulum'. Est autem haec subiectio necessaria ad tollendam superbiam, ad inducendam verecundiam et reverentiam, ad firmandam patientiam, ad tenendam oboedientiam. Superbia claudit oculos et tumidos efficit, quibus clausis et tumefactis veritatis radios mentis oculus non agnoscit".

58 Cf. Pseudo-Boethius, De disciplina scolarium, 6, ed. Weijers (nt. 53), 124, 18-19; 125, 19-126, 3: „Tenetur quoque doctor esse eruditus, mansuetus, rigidus, antiquus, nec negligens, non arrogans. [...] Non arrogans $[. .$.$] quoniam arrogantis magistri sedulitas fideliter numquam instruxit hominem. Hii sunt qui quod$ sciunt bumiliter docere contempnunt et quod recte sapiunt recte ministrare nequeunt, quoniam in fastigio positi fastigio merencium mentes despiciunt nec in datorem sciencie causam referunt, sed propriam in se respiciunt excellenciam".

59 Cf. ibid.

60 Cf. Compendium de virtute humilitatis, 2, in: Bonaventurae Opera omnia, vol. 8 (nt. 27), 658662, hier 659. „Valet etiam ad eam per hoc, quod vult magis esse discipula quam magistra et plus divinae 
Der Rekurs auf die Idee einer nicht-menschlichen Quelle des Wissens und auf die Notwendigkeit, eher an der göttlichen Autorität als an der menschlichen Vernunft festzuhalten (inhaerentia), klingt in der Tat in der topischen Benutzung der humilitas-Rede an, um an die ständige menschliche geistliche Abhängigkeit, Unvollkommenheit und Fehlbarkeit zu erinnern ${ }^{61}$.

Das Motiv der humilitas als erforderlicher Eigenschaft des magister kommt interessanterweise als polemische Waffe bei einigen Autoren aus dem universitären Milieu des 13. Jahrhunderts auf, um sich gegen die angebliche Arroganz einiger Kollegen zu positionieren ${ }^{62}$. Catherine König-Pralong hat insbesondere der Figur des Weltklerikers Gottfried von Fontaines und des Franziskaners Roger Bacon Aufmerksamkeit geschenkt, die sich durch unterschiedliche Ziele und auf verschiedene Weise als Gegenspieler innerhalb des akademischen Umfeldes von Paris profilierten ${ }^{63}$. Für beide Autoren wirkte die Berufung auf eine demütige Haltung als Gegengift gegen die erklärte Blasiertheit derjenigen Intellektuellen, die von sich selber und ihrer sozialen Klasse ein unangemessenes Bild zu liefern versuchten ${ }^{64}$. Interessanterweise aber hatten auch die Adressaten dieser Kritik - hier sind jeweils Thomas von Aquin und Heinrich von Gent zu nen-

sententiae inhaerere quam opinioni aut rationi propriae; et ideo nulli opinioni bumanae vel propriae pertinaciter inhaeret, divinas autem sententias reverentissime colit".

61 Im Compendium wird die Demut als mater omnis veritatis bezeichnet, indem sie „existimat enim omnia, prout sunt", cf. ibid., 658; siehe auch ibid., 659: „Ex praedictis autem patet quoque, bumilitatem sibi totum cor colligere et facere ipsum infra se stabiliter assistere et inhabitare; quia per intellectum nibileitatis suae et omnis creaturae ab omni vanitate speciei creatae mentem abstrabit, abstrabendo simplificat et depurat, et conspectui lucis aeternae sive entitati primae et summae animam reddit mundam, serenam, liberam, perviam et propinquam, eo quod nibil sibi ascribit nec in aliquo sibi innititur. Semper secum fert intimum sensum vel intellectum principalium operum Dei, scilicet creationis, reparationis et aeternae glorificationis".

62 Die polemische Kraft des humilitas/superbia-Topos im akademischen Kontext kann in ähnlicher Weise analysiert werden, wie das Antoine Destemberg in Bezug auf die Gattung der exempla neuerdings getan hat; diese exempla wurden in Dominikaner-Sermones aus der Mitte des 13. Jahrhunderts im negativen oder - eher seltener - positiven Sinn eingesetzt, um ein Bild der universitären magistri zu entwerfen. Destemberg hat neun Kategorien identifiziert, die die Hauptmotive der exempla veranschaulichen; unter diesen ist diejenige des Lasters der vana gloria eine der am meisten wiederkehrenden: „Une quarantaine d'exempla - pour 23 récits différents - , soit près du tiers de notre corpus, associe les universitaires à cette forme de péché d'orgueil qu'est la vana gloria [...] Pour les prédicateurs mendicants, la vaine gloire apparait comme le péché oppose à la vertu d'humilité don't ils se parent; son recours est d'autant plus pertinent pour dépeindre l'image des universitaires“, cf. A. Destemberg, L’honneur des universitaires au Moyen Âge. Étude d'imaginaire social, Paris 2015, 67-74, hier 70-71.

63 Cf. C. König-Pralong, Le bon usage des savoirs. Scolastique, philosophie et politique culturelle, Paris 2011.

64 Bezüglich der Darstellung und Selbstdarstellung der Meister aus einer vergleichenden Perspektive cf. I. P. Wei, The Self-Image of the Masters of Theology at the University of Paris in the Late Thirteenth and Early Fourteenth Centuries, in: The Journal of Ecclesiastical History 46 (1995), 398-431; id., From Twelfth-Century Schools to Thirteenth-Century Universities. The Disappearance of Biographical and Autobiographical Representations of Scholars, in: Speculum 86 (2001), 42-78 und neuerdings id., Intellectual Culture in Medieval Paris. Theologians and the University, c. 1100-1330, Cambridge 2012. 
nen - von derselben Topik, wenn auch mit anderen Ergebnissen, Gebrauch gemacht.

Vom scholastischen akademischen Umfeld grenzt sich Roger Bacon in diversen Schriften entschieden ab. Auf der Grundlage einer Wissensauffassung, gemäß der der Fortschritt im Lernen notwendig in einer gewissen Sittlichkeit verankert ist - in dem Sinne, dass die Verderbtheit der Menschen in studio notwendigerweise zu einer Verderbtheit der Menschen in vita führt ${ }^{65}-$, kritisierte Bacon im ,Compendium philosophiae', das um 1270 verfasst wurde, die intellektuellen Praktiken an der Universität Paris. Die corruptio seiner Zeit, behauptete der Franziskaner, bestünde unter anderem in dem Hochmut jener Kollegen, die sich selber als Meister der Theologie wähnten, ohne einem doctor im Studium der Philosophie gefolgt zu sein. Als Paradigma der unrechtmäßigen intellektuellen Arroganz galten für Bacon Albertus Magnus und Thomas von Aquin, welche, ,praesumpserunt in ordinibus investigare philosophiam per se sine doctore; ita quod facti sunt magistri in theologia et philosophia antequam fuerunt discipuli; et ideo regnat apud eos error infinitus"66. Die praesumptio beider Dominikaner ließe sich in ihrer Selbsterhebung zur Rolle des Meisters erkennen, ohne eine angemessene Ausbildung bekommen zu haben. Als Hindernis (impedimentum) des Fortschritts im Studium nannte Bacon in derselben Schrift die superbia als peccatum, die wesentlich der idealen Haltung des Schülers (discipulus) entgegengesetzt ist:

„Nam superbus non dignatur suam confiteri ignorantiam, nec se humiliare aliis, quod oportet fieri in discipulo. Quia praeesse desiderat, fit odiosus omnibus, nec recipitur in gratiam aliorum. Sed homo per se non potest proficere a juventute, et ideo cum aliis displiceat nec potest in studio proficere ullo modo. Et specialiter impedit superbia, quia superbus despicit alios, et aliorum scientias, et omnia reprobat quae ignorat ${ }^{\text {"67. }}$.

Ein gewisser Pessimismus im Hinblick auf die menschliche intellektuelle Neigung begleitete die Überlegungen Bacons. Als Folge der Erbsünde beugt die Unwissenheit den Menschen von seiner Geburt an, und selbst wenn jemand reif genug ist, um von seiner Vernunft Gebrauch zu machen, mache er sich nur aufgrund der Autorität Übergeordneter - Eltern oder Lehrer - auf die Suche

65 Cf. Roger Bacon, Compendium studii philosophiae, 1, in: Rogeri Bacon Opera quaedam hactenus inedita, vol. 1, ed. J. S. Brewer (Rerum Britannicarum Medii Aevi Scriptores. Rolls Series 15), London 1859 [Neudruck Nendeln/Liechtenstein 1965], 393-519, hier 402: „[...] impossibile est quod sapientia stet cum peccato, sed virtus perfecta requiritur ad eam, ut inferius demonstrabo. Sed pro certo si tantum de sapientia existeret, quantum apparet, non fieret ista in mundo. Nunc autem verissimum est, quod totum studium est in fine corruptionis a quadraginta annis, licet procurante Diabolo habeat infinitam apparentiam. Et homines corrupti in studio corrumpuntur in vita de necessitate". Cf. dazu König-Pralong, Le bon usage des savoirs (nt. 63), 137.

66 Cf. Roger Bacon, Compendium studii philosophiae, 5, ed. Brewer (nt. 65), 426. Cf. auch ibid., 429: „Nam numquam in aliqua aetate inventa fuit aliqua scientia, sed a principio mundi paulatim crevit sapientia, et adbuc non est completa in hac vita; propter quod infinita superbia invasit istos ordines, quod praesumunt docere antequam discant; et necesse est quod doctrina eorum sit in fine corruptionis".

67 Roger Bacon, Compendium studii philosophiae, 2, ed. Brewer (nt. 65), 408. 
nach der Weisheit ${ }^{68}$. Der Hochmütige aber weigert sich, die eigene Ignoranz einzusehen, und sich dem anderen zu öffnen. Er ist neidisch, unruhig und cholerisch, und dabei mangelt es ihm an Kommunikativität. Dadurch dass er gegenüber den anderen verschlossen ist, lässt der Hochmütige das lumen sapientiae nicht $\mathrm{zu}$; er ist nicht aufnahmefähig für die Weisheit ${ }^{69}$. Eine gewisse Ungeduld kennzeichnet ferner den Zornigen, der ,ex impetu spiritus praesumit de conclusione, antequam possit de praemissis probationibus judicare ${ }^{670}$. Es handelt sich um dieselbe Ungeduld, so könnte man glossieren, die Albert und Thomas dazu gebracht hatte, sich unverdient und frühzeitig als Autoritäten zu präsentieren. Wie KönigPralong gezeigt hat, werden die magistri scholastici von Bacon im ,Opus Maius“ sogar als Paradigmen des Hochmutes dargestellt und als negative Modelle des Nicht-Wissens oder des fiktiven Wissens ausgelacht ${ }^{71}$.

Gegen die Auffassung des Theologen als jenes erhabenen Wissenschaftlers, der eine direkte und übernatürliche Einsicht in die Glaubensprinzipien dank einer besonderen Auserwählung Gottes besitzt, richtete sich bekanntlich Gottfried von Fontaines in seinem, Quodlibet` IX, q. 2072. Dem nicht namentlich erwähnten Adressaten des Angriffes, Heinrich von Gent, warf er vor, der Theologie „plus dare [...] quam ei convenire possit"73. Die heinrichsche Rechtfertigung

68 Cf. Roger Bacon, Compendium studii philosophiae, 2, ed. Brewer (nt. 65), 404-405: „Omnis quidem homo scit per experientiam in se et in aliis, licet dissimulet caecitate mirabili, et negligat, quod homo totus est plena ignorantia et errore ab ipsa nativitate, ita quod etiam cum pervenit ad annos discretionis, et deberet uti ratione, et vivere in omnibus secundum vires ejus, repugnat omni rationi sicut brutum animal; ita quod nibil sapientiale quaereremus, nisi parentes et magistri et caeteri majores nos cogerent ad vias aliquas sapientiae; et tum ita rebelles sumus, quod aut nibil aut parum proficimus infra triginta primos annos".

69 Cf. Roger Bacon, Compendium studii philosophiae, 2, ed. Brewer (nt. 65), 408: „Invidus similiter qui alios odit, et dolet de utilitate proximi, necessario est odiosus aliis; et ideo nec communicat aliis, nec alii communicant ei, propter quod proficere non potest, praecipue cum ipsa invidia ex sua proprietate facit animum marcescere, et tabescere, et putrefieri spiritualiter, et deficere in se ipso; et ideo vires non habet anima invidiosa, nec potest ad ardua opera sapientiae penetrare. Similiterque sicut superbo non placent aliena, sed his detrabit, et reprobat ea quantumcunque sint dignissima, propter quod invidus ex sua proprietate quamcunque sapientiam in aliis despicit et annibilat, et ideo malitia sua non permittit eum lumen sapientiae recipere, sed blasphemat omnia quae ignorat".

70 Ibid., 408-409. In diesem Kontext verweist Bacon auf Platon: „Unde praeceptum fuit a Platone, quod iracundus non fiat discipulus sapientiae, nec studio ejus vacet, quoniam proficere non potest, eo quod animum semper habet turbatum, nec potest aliquid examinare veraciter, sed ex impetu spiritus praesumit de conclusione, antequam possit de praemissis probationibus judicare. Haec sententia Platonis in libro De Proportionibus et Proportionalitatibus recitatur".

71 Cf. König-Pralong, Le bon usage des savoirs (nt. 63), 140 sqq.

72 Cf. Gottfried von Fontaines, Quodlibet IX, q. 20, ed. J. Hoffmans (Les Philosophes Belges. Textes et Études 4), Louvain 1924, 282-293. Cf. König-Pralong, Le bon usage des savoirs (nt. 63), 105-123. In Bezug auf Heinrich von Gent und Gottfried cf. auch P. Porro, La teologia a Parigi dopo Tommaso. Enrico di Gand, Egidio Romano, Goffredo di Fontaines, in: I. Biffi/ C. Marabelli (eds.), Rinnovamento della ,via antiqua'. La creatività tra il XIII e il XIV secolo (Figure del pensiero medievale 5), Milano-Roma 2009, 165-262.

73 Gottfried von Fontaines, Quodlibet IX, q. 20, ed. Hoffmans (nt. 72), 287: „Sed isti plus videntur dare theologiae quam ei convenire possit, non propter defectum scibilis quod est perfectissimum in entitate et secundum se maxime cognoscibile, sed propter defectum nostrae infirmitatis quae etiam non patitur quod durante statu viae possumus per rationem efficacem attingere ad talem evidentiam, quia excluderet fidem quae est de ratione huiusmodi scientiae“. 
des außerordentlichen epistemischen Status des magister theologiae, die einerseits von den auctoritates sanctorum, andererseits von der nicht weiter überprüfbaren Anwesenheit einer besonderen göttlichen Erleuchtung herrühre, bezeichnete Gottfried mit den Adjektiven irrationabile und verecundum ${ }^{74}$. Dabei forderte er ein bescheidenes Bekenntnis zur eigenen Mangelhaftigkeit von demjenigen, der sich für einen Meister halten will:

"Quomodo ergo honeste potest dicere magister huiusmodi scientiae hanc scientiam esse veram scientiam solum ex hoc, quia sancti videntur hoc dicere? Ex hoc enim solum poterit vere dicere se credere hoc esse verum; sed nesciet ita esse, quia etiam nesciet se habere talem notitiam, quia in nulla conclusione hoc potest ostendere. Debet enim ille, qui buiusmodi scientiae reputat se esse magistrum, scire se competenter habere evidentiam notitiae quae in hac scientia convenienter est nata haberi; licet ex bumilitate debeat dicere se illam habere minus sufficienter quam haberi possit $[\ldots]^{\text {“75 }}$.

Das Motiv der infirmitas des menschlichen Geistes taucht ebenso bei Gottfried zusammen mit der Idee auf, dass niemand in diesem Leben eine Wissenschaft (scientia) des Göttlichen erreichen kann. Umso weniger kann ein magister eine Garantie im Hinblick auf den Besitz eines solchen Wissens von sich selber aus liefern ${ }^{76}$.

Interessanterweise hatte auch Thomas in seiner 1270 gehaltenen determinatio zur ,Quaestio quodlibetalis“ „Utrum liceat quod aliquis pro se petat licenciam in theologia docendi" die Gefahr der praesumptio im magistralen Bereich berührt, wenn auch mit unterschiedlichen Zielen ${ }^{77}$. Der Dominikaner gründete seine Antwort auf die Unterschiede zwischen der cathedra pontificalis, für die es nicht ehrenhaft sei, die licentia für sich selber zu verlangen, und der cathedra magistralis, für die hingegen die Möglichkeit eingeräumt werden müsse, die licentia docendi zu fordern. Die licentia docendi, so Thomas, füge der betroffenen Person keine neue eminentia, sondern lediglich die Gelegenheit hinzu, das eigene, schon vorhandene Wissen weiterzuvermitteln ${ }^{78}$. Und diese eminentia scientiae, die zum Erlangen des Lehrstuhles erforderlich sei, betreffe die "perfectio hominis secundum se ipsum "79. Eine unabdingbare Bedingung für eine solche petitio sei daher, dass im Meister ein adäquates Wissen tatsächlich vorhanden sei:

„Cum autem ille qui accipit licenciam ad cathedram magistralem accipiat solam oportunitatem communicandi quod habet, petere huiusmodi licenciam, quantum est in se, nullam videtur turpitudi-

74 Cf. ibid., 289.

75 Ibid.

76 Derisibiles wären für Gottfried jene sogenannten magistri, die die Theologie als ,vere et proprie dicta scientia“ bezeichnen, sie aber nicht besitzen, „qui tanto tempore in ea laboraverunt“. Als ein figmentum nannte er weiterhin die Einbildung jener magistri, die sich eine besondere und exklusive Erleuchtung Gottes vorbehielten, cf. Gottfried von Fontaines, Quodlibet IX, q. 20, ed. Hoffmans (nt. 72), 289-290.

77 Cf. Thomas von Aquin, Quodlibet III, q. 4, a. 1, ed. Commissio Leonina (Opera omnia 25/2), Roma-Paris 1996, 251-253. Cf. dazu König-Pralong, Le bon usage des savoirs (nt. 63), 64-65. S. auch E. Marmursztejn, L'autorité des maittres. Scolastique, normes et société au XIII ${ }^{\mathrm{e}}$ siècle, Paris 2007, 33-34.

78 Cf. Thomas von Aquin, Quodlibet III, q. 4, a. 1, ed. Leonina (nt. 77), 252.

79 Ibid. 
nem continere, quia communicare aliis scienciam quam quis habet laudabile est et ad caritatem pertinens [...] Potest tamen turpitudinem continere ratione presumptionis, que esset si ille qui non est ydoneus ad docendum peteret docendi officium" "80.

Das vitium der praesumptio in Bezug auf die Evaluation des eigenen Wissens kann aber, so Thomas weiter, gerade dadurch vermieden werden, dass das Kriterium für ein sicheres Urteil über das eigene Wissen dem Subjekt selber innewohnt („Nam scienciam, per quam aliquis est ydoneus ad docendum, potest aliquis scire per certitudinem se habere") ${ }^{81}$.

Ein strategischer Rekurs auf die Topik der Bescheidenheit ist ebenso in der ausführlichen Beschreibung des doctor sowie des auditor der akademischen Theologie zu lesen, der Heinrich von Gent eine beachtliche Anzahl an disputierten ,Quaestiones' in der Anfangszeit seiner universitären Karriere, das heißt wahrscheinlich schon vor dem Jahr 1276, widmete ${ }^{82}$. Als Eigenschaft des „proprius et conveniens auditor" der scientia theologica erwähnte Heinrich die Fähigkeit, sich die tiefen Wahrheiten jenes Wissens anzueignen und zwischen den bene dicta und den contraria unterscheiden zu können ${ }^{83}$. Den natürlichen Begabungen füge sich aber auch als erforderliches Element eine solide und vollständige Vorbereitung in den weltlichen Disziplinen hinzu, um die Geheimnisse der theologischen Wissenschaft sondieren zu können, und das erlangte Wissen an das Volk weiterzugeben ${ }^{84}$. Dabei sollte der auditor theologiae nicht nur bei der praedicatio, sondern auch bei der lectio zuhören, um sich der Gefahr der praesumptio zu entziehen:

„Dicendum quod tales omnino debent esse auditores huius scientiae, non solum per praedicationem, sed etiam audiendo eam per lectionem, dummodo praedicatione non potuerint sufficienter instrui ut sint idonei alios docere secundum gradum status sui. Aliter enim exponerent se discrimini ex praesumptione" 85 .

Interessanterweise kehrt bei Heinrich von Gent zusammen mit der Fürsprache für eine geeignete Gelehrsamkeit der doctores das Zitat aus ,De disciplina scholarium" „Non enim erit magister qui non se novit esse discipulum" 86 wieder. Das weltliche Wissen bereitet, dem flandrischen Autor zufolge, den Geist und die Scharfsinnigkeit des Schülers vor und beide werden als Propädeutik zur Öffnung für ein höheres Wissen betrachtet, das den Glauben als notwendige Vorausset-

80 Ibid., 253, 79-92.

81 Ibid., 253. Zu Thomas' Auffassung der bumilitas cf. A. Kenny, St Thomas Aquinas on the Virtue of Humility, in: J. Beneš/P. Glombíček/V. Urbánek (eds.), Bene Scripsisti... Filosofie od

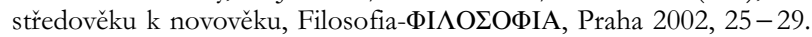

82 Cf. dazu A. J. Minnis, The Accessus Extended: Henry of Ghent on the Transmission and Reception of Theology, in: M. D. Jordan/K. Emery Jr. (eds.), Ad litteram. Authoritative Texts and Their Medieval Readers (Notre Dame Conferences in Medieval Studies 3), Notre DameLondon 1992, 275-326; König-Pralong, Le bon usage des savoirs (nt. 63), 69-104.

83 Cf. Heinrich von Gent, Summa quaestionum ordinariarum, 12, q. 2, ed. I. Badius, vol. 1, Parisiis 1520 [Neudruck St. Bonaventure, NY 1953], 84ㄷ․

84 Cf. Heinrich von Gent, Summa quaestionum ordinariarum, 12, q. 6, ed. Badius (nt. 83), $88^{\text {r V. }}$

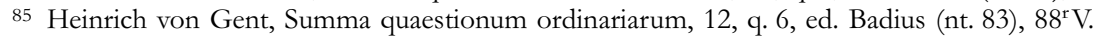

86 Heinrich von Gent, Summa quaestionum ordinariarum, 12, q. 6, ed. Badius (nt. 83), $88^{r} \mathrm{X}$. 
zung und als ein besonderes göttliches Licht als auserwählte Hilfe hat ${ }^{87}$. Neben den Frauen und den Jungen, die nach Heinrich über diesen Scharfsinn nicht verfügen, ist auch derjenige von dem Zugang zu der theologischen Wissenschaft ausgeschlossen, der lediglich der natürlichen Vernunft zustimmt. Der Schüler, der in den weltlichen Wissenschaften belehrt ist, muss sich in einer bescheidenen Haltung der Theologie annähern (,humilis et ut parvulus ad eam veniat ") ${ }^{88}$. Der Verweis auf die humilitas fungiert dabei als Gelegenheit, um die Distanz der Theologen zu den philosophi zu verdeutlichen ${ }^{89}$.

Sind einmal die Voraussetzungen für das Studium der Theologie erfüllt, so muss der Theologe sich nicht für seine Wissenschaft schämen. Es ist bloß im Bereich der natürlichen Vernunft, dass der Mensch seine eigene infirmitas erkennen muss ${ }^{90}$. Der Meister in Theologie ist derjenige, der schon über eine Expertise verfügt und der sich bereits für das Übernatürliche geöffnet hat. Ihm kommt also die Demut nicht als proprium zu. Heinrich von Gent beantwortete die Frage, ob eine Frau als „doctor huius scientiae“, das heißt der theologischen Wissenschaft, hervortreten könne, negativ und listete zugleich vier Voraussetzungen für das „docere ex officio“ auf, unter denen die Bescheidenheit keine Rolle spielt: 1. Festigkeit/Standhaftigkeit in der Lehre (docendi constantia); 2. Wirksamkeit (exequendi efficacia); 3. Autorität des Lehrers (docentis auctoritas); 4. Lebhaftigkeit der Rede (sermonis vivacitas) $^{91}$. Die simplicitas und Ungelehrtheit werden lediglich in Bezug auf die ersten Lehrer der Theologie, die Apostel, positiv konnotiert. Die intellektuelle Bescheidenheit oder Unwissenheit, die die Anfänge der christlichen Tradition kennzeichnete, ist für Heinrich von Gent ein historisches Faktum, das nicht mehr nachgeahmt werden kann oder soll ${ }^{92}$.

Für beide, Thomas von Aquin und Heinrich von Gent, ist also das öffentliche, offiziell anerkannte docere mit dem vollkommenen Besitz einer scientia ver-

87 Zur heinrichschen Lehre des besonderen theologischen Lichtes cf. neuerdings A. Arezzo, Lumen medium: Enrico di Gand e il dibattito sullo statuto scientifico della teologia (Biblioteca filosofia di Quaestio 21), Bari 2014.

88 Heinrich von Gent, Summa quaestionum ordinariarum, 12, q. 5, ed. Badius (nt. 83), 87º.

89 Cf. ibid.

90 Cf. Heinrich von Gent, Summa (Quaestiones ordinariae), 4, q. 5, ed. G. A. Wilson (Opera omnia 21), Leuven 2005, 296, 184-297, 198.

91 Heinrich von Gent, Summa quaestionum ordinariarum, 11, q. 2, ed. Badius (nt. 83), 77º: „Dicendum igitur ad istam propositam quaestionem et consimiliter ad alias quod docere potest aliquis ex officio vel ex beneficio. Ut autem aliquis possit docere ex officio quatuor in eo requiruntur, duo respectu doctrinae et duo respectu illorum quibus dispensanda est doctrina. Primum est docendi constantia, ne de facili a veritate cognita doctor se divertat. Secundum est exequendi efficacia, ne per infirmitatem ab opere desistat. Tertium est docentis auctoritas, ut auditores sibi credendo obediant. Quartum est sermonis vivacitas, ut vitia corrodendo ad virtutes impellat". Cf. dazu und zu der parallelen Auffassung des Thomas bezüglich der Rolle der Frau in der öffentlichen Lehre E. Gössmann, Anthropologie und soziale Stellung der Frau nach Summen und Sentenzenkommentaren des 13. Jahrhunderts, in: G. Vuillemin-Diem/A. Zimmermann (eds.), Soziale Ordnungen im Selbstverständnis des Mittelalters, 1. Halbband (Miscellanea Mediaevalia 12/1), Berlin-New York 1979, 281 -297, hier insbes. 295.

92 Cf. dazu König-Pralong, Le bon usage des savoirs (nt. 63), 78-79. Einige Überlegungen zum Thema der Bescheidenheit bei Heinrich von Gent habe ich hier geboten: S. Negri, Veritatem bumiliter investigare. Sul ruolo dell'umiltà in Enrico di Gand, in: Quaestio 15 (2015), 607-617. 
knüpft, derer sich der magister bewusst ist. Dieser Status ist für beide Autoren epistemisch beschreibbar, objektiv erfassbar und unbestreitbar. Es ist kein $\mathrm{Zu}-$ fall, dass der Faktor der Demut keine Rolle in den genuin epistemisch ausgerichteten Analysen des Prozesses des Lehrens und Lernens schlechthin spielte, die bekanntlich zunächst Thomas von Aquin und dann Heinrich von Gent in der 11. ,Quaestio disputata“ „De veritate“ und im ersten Artikel der ,Summa quaestionum ordinariarum' skizziert hatten ${ }^{93}$. Ein epistemisches Kriterium - die bloße Potentialität oder die Aktualität im Besitz einer Wissenschaft (scientia) - dient dazu, die Notwendigkeit des kontinuierlichen Bekenntnisses der eigenen Niedrigkeit im Wissensbereich zu entschärfen. Die Sicherheit über den Besitz einer genügenden Wissenschaft wird somit in allen genannten Paradigmen zum Garanten gegen die Laster der praesumptio, der sich die Bescheidenheit entgegenstellt. Wo aber und von wem diese Garantie eingeholt werden soll und kann, bleibt als unvermeidlicher Unterscheidungsfaktor bestehen.

\section{Zurück zur Topik der bumilitas \\ Universitäre Statuten und die vorgeschriebene reverentia}

Mehrfach hat Dilwyn Knox die These vertreten, dass die mittelalterliche christlich, monastisch und kirchlich konnotierte Idee von Disziplin das neuzeitliche, säkularisierte Konzept der Disziplin des civis und der Zivilisation prägte. Es handelt sich nach Knox um die Idee einer Balance zwischen einer geistigen und körperlichen, innerlichen und äußerlichen Haltung, die als Voraussetzung eines guten Verhaltens diente ${ }^{94}$. Die Aufforderung zur modestia identifizierte Knox als architektonisches und programmatisches Element in jenen Texten, die sich mit dem guten Benehmen - des Novizen, des Klerikers und dann des guten Bürgers - beschäftigten. Auf die einzigartige Aneignung der Bescheidenheit durch christliche mittelalterliche Autoren ging Knox ein, um zu zeigen, wie die christliche Pädagogik der modestia zur Grundlage des „Laienideals“ der guten Manieren in der „ganzen christlichen Latinität", sowohl der katholischen als auch der protestantischen, wurde ${ }^{95}$. Eine gewisse Persistenz und Universalität im Vorkommen ähnlicher Bilder stellte er in unterschiedlichen Quellentypologien aus

93 Zu einigen noetischen Aspekten der betroffenen Quästionen cf. W. Goris, De magistro - Thomas Aquinas, Henry of Ghent and John Duns Scotus on Natural Conceptions, in: The Review of Metaphysics 66 (2013), 435-468.

94 Cf. D. Knox, Disciplina: le origini monastiche e clericali del buon comportamento nell'Europa cattolica del Cinquecento e primo Seicento, in: Prodi (ed.), Disciplina dell'anima (nt. 40), $63-$ 99. Cf. auch id., Disciplina. The Monastic and Clerical Origins of European Civility, in: J. Monfasani/E. F. Rice (eds.), Renaissance Society and Culture. Essays in Honor of Eugene F. Rice, Jr., New York 1991, 107-135 und id., Disciplina del corpo e dell'anima. L'eredità del Medioevo, in: C. Casagrande/S. Vecchio (eds.), Anima e corpo nella cultura medievale. Atti del V Convegno di studi della Società Italiana per lo Studio del Pensiero Medievale, Venezia, 25-28 settembre 1995 (Millennio Medievale 15, Atti di Convegni 3), Firenze 1999, 277-287.

95 Cf. Knox, Disciplina: le origini monastiche e clericali (nt. 94), 77. 
dem 15. und 16. Jahrhundert fest, zu denen auch die ,Constitutiones' der Jesuiten gezählt werden ${ }^{96}$.

Die Vorschrift der modestia ist aber auch in den Statuten einiger universitärer Institutionen zu beobachten, die in Mitteleuropa im 14. und 15. Jahrhundert entstanden sind ${ }^{97}$. Dadurch dass die mittelalterlichen Universitätsstatuten die Gemeinschaft der Schüler und der Meister mit Normen zur Erlangung der akademischen Grade regulierten, setzten sie objektive Kriterien, um die Quantität des vorhandenen Wissens zu definieren. Dabei schrieben sie auch das geeignete Verhalten der Mitglieder vor, das zur Integrität der universitas beitragen sollte.

Als Grundregel des akademischen Zusammenlebens schrieben zum Beispiel die ersten Statuten der Universität Tübingen (1477) den Studierenden neben einer gewissen Sittlichkeit in der Unterhaltung und Bekleidung vor, sich gegenüber den Übergeordneten, das heißt den Doktoren und den Lehrern, mit reverentia und oboedientia zu verhalten. Das anständige Benehmen der Schüler (studentes) sollte deren Ehre und damit - oder noch davor - den Ruhm der Universität selbst vermehren:

„Hortandi sunt deinceps studentes primo ad mores, ut se honeste regant in conversatione et vestitu, et eius superioribus, videlicet doctoribus et magistris, debitam reverentiam et oboedientiam impendant, et cum aliis pacifice vivant, neminem ledendo, in bonis exterioribus aut corpore vel verbo, signo vel facto, sed omnibus taliter se exbibeant, ut ex hoc bona universitatis crescat fama et honor eorundem laudabilis" ${ }^{\prime 98}$.

Die drei Jahre später verfassten Statuten der facultas theologica derselben Universität befahlen, dass der Promovierende ${ }^{99}$, der in die aula für die feierliche Disputation eintrat, seine Knie beugen und den Lehrern Ehrfurcht schwören sollte ${ }^{100}$. Ähnliche Vorschriften kann man in den ersten Statuten der Artes-Fakultät der Universität Freiburg finden, in denen unter der Rubrik „De moribus“ festgelegt

96 Cf. ibid.

$97 \mathrm{Zu}$ den Universitäten in Mitteleuropa im Spätmittelalter seien hier paradigmatisch genannt: J. Miethke, Die Welt der Professoren und Studenten an der Wende vom Mittelalter zur Neuzeit, in: K. Andermann (ed.), Historiographie am Oberrhein im späten Mittelalter und in der frühen Neuzeit (Oberrheinische Studien 7), Sigmaringen 1988, 11-33; F. Rexroth, Deutsche Universitätsstiftungen von Prag bis Köln. Die Intentionen des Stifters und die Wege und Chancen ihrer Verwirklichung im spätmittelalterlichen deutschen Territorialstaat (Beihefte zum Archiv für Kulturgeschichte 34), Köln-Weimar-Wien 1992; die Aufsätze von W. J. Courtenay, R. C. Schwinges, K. Wriedt und F. Rexroth im Band W. J. Courtenay/J. Miethke (eds.), Universities and Schooling in Medieval Society (Education and Society in the Middle Ages and Renaissance 10), Leiden-Boston-Köln 2000; R. C. Schwinges, Zur Professionalisierung gelehrter Tätigkeit im deutschen Spätmittelalter, in: H. Boockmann/L. Grenzmann/B. Moeller/M. Staehelin (eds.), Recht und Verfassung im Übergang vom Mittelalter zur Neuzeit, II. Teil (Abhandlungen der Akademie der Wissenschaften in Göttingen 239), Göttingen 2001, 473-493, mit ausführlichen bibliografischen Hinweisen.

98 Urkunden zur Geschichte der Universität Tübingen aus den Jahren 1476 bis 1550, Tübingen $1877,53$.

99 Der promovendus ist derjenige, der die licentia in theologia bekommen soll; cf. ibid., 260.

100 Cf. ibid., 261: „Primo aulandus flectat genua et iuret reverentiam magistrorum“. 
wurde, dass die Studierenden, welche sich respektlos gegenüber den Dozierenden benahmen, nicht zu den Prüfungen zugelassen werden sollten, selbst wenn sie im Studium erfolgreich waren ${ }^{101}$. Die den mores im Statut zugesprochene Bedeutung für die offizielle Erlangung der Lizenz verrät die Idee, dass der Lehrerfolg (das ,proficere in scientiis") nicht von den guten Sitten notwendigerweise abhängt. Die guten Sitten sind aber für die Erlangung eines Grades unabdingbarr $^{102}$.

Schon die im Jahre 1365 verfassten Statuten der Artes-Fakultät von Wien waren bezüglich des guten Verhaltens der Studierenden ganz explizit. Dem Titulus VI „De vita et moribus scolarium facultatis artium“ entnimmt man, dass Unterwerfung unter die Lehre des Meisters, Mäßigkeit, Hartnäckigkeit, Bescheidenheit hier mit dem Adverb modeste ausgedrückt -, Ruhe und Gehorsamkeit zu einer guten Beschaffenheit des discipulus gehören ${ }^{103}$. Neben dem Verbot, während der Nacht fragwürdige tabernae zu besuchen, Streit anzufangen oder gegenüber den Mitgliedern der Universität beleidigende Pamphlete zu verfassen, wurde es zudem im selben statutarischen Paragrafen den Studierenden untersagt, sich in offiziellen Angelegenheiten unbegründet über die Autorität der Magister hinwegzusetzen:

„Item nullus scolarium nostre facultatis se erigat de tanto, quod presumat se locare super magistros nostre facultatis in aliquo actu publico etiam totius universitatis, scilicet in sermonibus, lectionibus, disputationibus et aliis consimilibus, cuiuscumque facultatis actus fuerit, nisi dignitas persone vel sui officii status id exposcat" 104 .

Das Verbot der praesumptio in den öffentlichen Akten trat im akademischen Wesen als Garant auf für die Einhaltung der Hierarchie der Status, in denen die soziale Rolle der dignitas persone vel officii etabliert wird.

Eine tiefgehende und breitere Analyse der Statuten der europäischen Universitäten als literarischer Texte wäre ohnehin erforderlich, um Schlussfolgerungen

101 Cf. H. Ott/J. M. Fletcher, The Mediaeval Statutes of the Faculty of Arts of the University of Freiburg im Breisgau (Texts and Studies in the History of Mediaeval Education 10), Notre Dame, IN 1964, 43: „Item quia deficientes in moribus, etsi proficiant in scientiis, constat plus deficere quam proficere, ideo facultas artium vult, ordinavit et statuit quod subsequentes non admittantur ad examen vel tentam baccalariatus vel magisterii: scilicet lusores, discoli, brigatores, infames, scandalosi, doctoribus vel magistris irriverentiam exbibentes, in vestibus indecentibus more rutbeorum seu armiferorum aut alias immorigerate et non scolastice se gerentes, turbatores pacis".

102 Wie kürzlich von Destemberg gezeigt worden ist, beschrieb Johannes Gerson am Anfang des 15. Jahrhunderts die Erlangung eines akademischen Grades als eine vielfache conversio für den Lizenziaten, cf. Destemberg, L'honneur des universitaires au Moyen Âge (nt. 62), 144-147.

103 Cf. A. Lhotsky, Die Wiener Artistenfakultät 1365-1497 (Österreichische Akademie der Wissenschaften. Philosophisch-historische Klasse 247/2), Wien 1965, 234: „Non modicum differt sic vel sic assuesci a iuventute, scilicet bene vel male. Bone etiam indolis est se flectere secundum doctrinam sui praeceptoris. Volumus igitur facultatis discipulos, sicut et docemus, fore morigeratos, mansuetos et pacificos et studiosus continuos et oboedientes magistris et suarum bursarum rectoribus. In lectionibus et disputationibus sine murmure, cachinno et sibilis et ululatibus, sed more virginum et constanter et modeste persistere a principio usque ad finem".

104 Ibid., 235. 
bezüglich der Vorschriften der Bescheidenheit im akademischen scholastischen Bereich zu ziehen ${ }^{105}$. In den hier analysierten Themenbereichen haben sich immerhin Bedeutungswandlungen angedeutet, die auf eine normierende Absicht im pädagogischen Bereich hinweisen. Betrachtet man wiederum einerseits jene regulierenden Diskurse, die sich mit der Totalität der vita eines Einzelnen in einer Gemeinschaft beschäftigen, das heißt den monastischen Regeln, andererseits die eben herangezogenen universitären Statuten, welche ebenso die Verhältnisse einer Gemeinschaft im Bereich des Wissensnachweises normieren, kann man Folgendes feststellen. Drückt sich in den monastischen Regeln die Vorschrift der bumilitas in einer ganzheitlichen Norm des praktischen Lebens aus, die Seele sowie Leib in einer aufsteigenden Verinnerlichung des Gebotes zu disziplinieren vermag, beschränkt sich die Anordnung der Mäßigung in den Statuten auf äußerlich unangemessene Verhaltensweisen. Das Element der Selbst-Erbauung und Selbst-Verwirklichung des Menschen als Mensch mittels der Disziplin der bumilitas sowie die effiziente und finale Ursächlichkeit der Liebe Gottes, die in der ,Regula' und in den betrachteten Texten Hugos von Sankt Viktor den Kern der Bescheidenheits-Lehre darstellen, sind in der erwähnten statutarischen Literatur als solche nicht zu finden. Dort bleibt die Mahnung zur Bescheidenheit auf dem Niveau der Vorschrift von objektiv feststellbaren Akten. Es ist kein Zufall, dass die Normen zur Unterwerfung in Verbindung mit den Regelungen bezüglich anständiger Kleidung der Studierenden auftauchen. Das Ziel, die Bescheidenheit zu praktizieren, ist dort einerseits immanent vorhanden - ein gemäßigtes Benehmen ist Voraussetzung, um die studentische Karriere zu vollenden -, andererseits wird es explizit mit dem guten Ruf der universitären Institution selbst verbunden. Die Bescheidenheit wird zur Norm der Sittlichkeit in der akademischen Gemeinschaft und zur Anerkennung der Autorität - und somit der Erhabenheit - der Meister und ihrer Grade ${ }^{106}$.

Alles in allem beinhaltet die Vorschrift und anvisierte Praxis der Bescheidenheit unter einer allgemeinen, formalen Perspektive in allen betrachteten Quellen mit Bezug auf eine Lehrende-Lernende-Beziehung die Konzepte der Mäßigung und Gehorsamkeit, der Stille und des Dienstes. Die Topik der Demut kehrt wieder in den räumlich konnotierten Bildern der Verbeugung des Schülers gegenüber dem Meister, der Untersagung, sich oberhalb des Meisters zu positionieren (se erigere), der Unterwerfung (submissio) und der zu vermeidenden praesumptio.

105 Mit Blick auf die Rolle der guten Sitten sowie der bumilitas für die Legitimierung der intellektuellen akademischen Gemeinschaft der Meister zu Beginn bemerkt Destemberg, L'honneur des universitaires au Moyen Âge (nt. 62), 322: „L'identité magistrale reposait sur une ambiguïté difficilement réductible: la virilité du maitre constituait le support physiologique de son intelligence, mais ne devait en rien s'exprimer dans ses moeurs. Dès le XII ${ }^{\mathrm{e}}$ siècle, les moeurs des maîtres et des étudiantes furent ainsi une pierre angulaire de leur identité sociale. Autour des principes chrétiens de l'humilité, de la foi et de la charité se structura un champ littéraire de la renommée scolaire, qui accompagna chronologiquement le premier mouvement d'institutionnalisation de l'Université, jusqu'au milieu du XIII ${ }^{\mathrm{e}}$ siècle“.

106 Cf. dazu Destemberg, L'honneur des universitaires au Moyen Âge (nt. 62), insbes. 93-94. 
Die Demut wirkt als Garant der Anerkennung und Rücksicht auf die Hoheit des Lehrenden, die ihrerseits auf der Niedrigkeit des Schülers basiert. Dabei scheint die Verknüpfung zwischen dem semantischen Feld der Lehre und demjenigen der Demut in den betrachteten Texten insofern wesentlich, als die Beziehung zwischen den im Lernprozess involvierten Figuren - magister und discipulus, doctor und studens - eine Rezeptivität und Aufnahmefähigkeit vonseiten des Schülers voraussetzt, welche die demütige Haltung gewährleistet.

Als notwendige Eigenschaft der Schüler scheint die Demut insbesondere in folgenden Kontexten aufzutauchen: 1. in jenen mittelalterlichen Diskursen, deren Gegenstand, Ton oder Ziel mit pädagogischen oder normativen Vorhaben verbunden sind, das heißt in jenen Texten, die Regeln für den discipulus vorschreiben, Empfehlungen für das Lehren und Lernen vermitteln und sich mit der Sittlichkeit des Wissens beschäftigen; 2. in den Diskursen, wo das betrachtete oder empfohlene Wissen einen Weisheitscharakter beinhaltet; 3. in Kontexten, in denen der Rekurs auf die Bescheidenheits-Topik als kritisches und polemisches Element in der Selbstdefinierung und Selbstabgrenzung der Magister diente. Die bumilitas ist daher eine Figur der Praktik des Wissens. Abgesehen aber von der Art des anzueignenden Wissens (eines praktischen Könnens und der Kenntnis einer Norm, einer Wissenschaft sowie einer Regel des guten Benehmens) fängt der Prozess der Wissensvermittlung mit einem Moment des Sich-Öffnens und der Verbeugung seitens des Schülers an, der durch einen von Demut gekennzeichneten Akt initiiert wird. Die Topik der humilitas taucht einmal im Kontext einer Anerkennung der Autorität des Meisters auf, ein anderes Mal als Einsicht in die eigene Unwissenheit, die als Antrieb zur Suche nach dem fehlenden Wissen wirkt, oder aber als Achthaben und als Verzicht auf den eigenen Willen. In der Gefügigkeit des Geistes und des Körpers drückt sich kurzum die Fähigkeit aus, von dem Lehrer oder von dem Wissen selber geformt und geprägt zu werden. Die Verbindung zwischen dem Einzelnen und dem Kollektiv spielt dabei eine zentrale Rolle, da jeder Schüler sich als Teil einer Gemeinschaft von Lernenden und Lehrenden profiliert, in der ein wechselseitiges Aufnahmeverfahren stattfinden soll, und in der die jeweiligen Positionen beibehalten werden sollen. Die Rücksicht auf die Hoheit ist die Kehrseite der Gleichsetzung aller derjenigen, die zu lernen versuchen. Konzipiert man Lehren und Lernen als einen einheitlichen epistemischen Prozess oder als soziale und politische Akte, so kann jene Aufnahmefähigkeit des Lernenden und des Lehrenden als notwendiges Fundament der erfolgreichen Vermittlung betrachtet werden. 
